\title{
Influence of Spherical and Pyramidical Dimples and Bumps on Airfoil Performance in Subsonic Flow
}

\author{
Zahra Mehtar ${ }^{1} \mathbb{D}$, Afaq Altaf ${ }^{1, *} \mathbb{D}$
}

\begin{abstract}
1.New York Institute of Technology - School of Engineering and Computing Sciences - Abu Dhabi - The United Arab Emirates. *Corresponding author: afaqaltaf@hotmail.com
\end{abstract}

\section{ABSTRACT}

In this study, surface features such as dimples and bumps are introduced to the surface of a NACA 0012 airfoil to study their effect on boundary layer separation, particularly at high angles of attack. Six modified airfoils were designed with dimples and bumps of spherical and pyramidical shapes. A computational fluid dynamics (CFD) analysis was conducted on these models at subsonic flow using Ansys Fluent. The analysis used the Shear Stress Transport $k-\omega$ turbulence model at a varying angle of attack (AOA) from 0 to $15^{\circ}$. The velocity contours and streamlines were generated. Also, the lift coefficient, drag coefficient and the lift-to-drag performance ratio were computed and analyzed. The results showed that all surface modifications led to delayed flow separation and flow recirculation. All surface modification also resulted in a decrease in drag at $15^{\circ}$. All designs, except pyramidical protrusions, increased the lift-to-drag ratio (L/D) performance at $15^{\circ}$. It was found that dimples are better than bumps and spherical features are better than pyramidical ones.

Keywords: Vortex; Airfoil; Aerodynamic Performance; CFD; Ansys Fluent.

\section{INTRODUCTION}

The aviation industry today is the safest and most efficient it has been since the first aircraft was invented. However, there is always room for improvement. A new area of interest is the study of texture on an aircraft wings. These textures, which can be raised or depressed, have shown to induce local vortices. These vortices increase the momentum of the boundary layer and decrease pressure drag. These conditions lead to a delay in boundary layer separation at higher angles of attack (AOA) and, consequently, increase the stall angle. Stall is a highly unfavorable phenomenon that reduces an aircraft performance and safety. Therefore, reducing the pressure drag is of great interest as it reduces fuel consumption, increases safety, and improves the overall performance of an aircraft.

Research to understand the effect dimples and protrusions have on the boundary layer separation and pressure drag of an airfoil is numerous and ongoing.

Hong and Asai (2017) studied the effect of various surface patterns on the performance of a soccer ball. The study was conducted in a wind tunnel and the forces acting on the ball were analyzed. They found that the dimpled balls performed better than the dimple-less balls for the subcritical Re number range while the dimple-less balls performed better in the supercritical range.

Received: Nov. 15, 2020 | Accepted: Mar. 10, 2021

Peer Review History: Single Blind Peer Review.

Section Editor: SungKi Jung 
Livya et al. (2015) investigated the influence of spherical, hexagonal, cylindrical, and square dimples and bumps on the performance of a NACA 0018 airfoil. They found that dimples performed better than bumps, although both performed better than the clean airfoil.

Ramprasadh and Devanandh (2015) conducted a computational fluid dynamics (CFD) analysis on the effect of dimples on the performance of a low aspect ratio modified inverse Zimmerman planform wing with a SELIG4083 cross-section. They found that the dimples create local vortices that aids in lift generation. They also found that more than five rows of dimples do not contribute significantly to the reduction of pressure drag and could increase skin friction drag. They also concluded that the strength of the vortices over the airfoil increases with the increase in the dimples depth and their proximity to each other.

Chalia and Bharti (2017) aimed to reduce drag, increase lift, and delay flow separation and stall by the introduction of spherical dimples and vortex generators over a NACA 2412 airfoil. They found that the performance of an airfoil with surface features is far superior to a clean airfoil. They also found that the skin friction drag is smaller for dimples. Vortex generators (bumps) increased the lift coefficient, but also increased the drag coefficient.

Saraf et al. (2017) analyzed a NACA 0012 airfoil that was altered by dimples. They conducted a CFD analysis for dimples located at four different positions along the airfoil. They found that for a dimple at $75 \%$ of chord length, the $C_{l}$ increased by $7 \%$, while $C_{d}$ decreased by $3 \%$. They concluded that the airfoil with the dimple at $75 \%$ chord is the best performing.

Bogdanović-Jovanović et al. (2012) carried out an experimental study to understand the flow behavior for a dimpled ball. They found that due to the dimples, the critical region of the sphere shifted towards a lower Re number, suggesting that the dimpled sphere has lower drag at lower speeds.

Hornea and Simion (2019) studied pressure distribution and airflow around a golf ball for six airspeeds. They found that a dimpled ball had better reactivity to sudden changes in airspeed. They found that the drag reduces as the airspeed increases for a dimpled ball and the drag is more predictable at higher speeds. The ball also had a better efficiency at greater speeds. They concluded that a dimpled surface reduced skin friction drag by creating a turbulent boundary layer, which acts as a lubricating mechanism. The tiny vortices created by the dimples act like small ball bearings that reduce the friction between the ball and air layers.

Zulkefli and MohdNur (2020) studied the effect of an inboard dimple, an outboard dimple, and a triangular vortex generator at $50 \%$ chord length on the flow around a NACA 4415 airfoil. They found that the lift is highest for a triangular vortex generator while the drag is lowest for the inward dimple and triangular vortex generator. The inward dimple has the best lift-to-drag ratio (L/D) performance from all modifications.

Mustak et al. (2015) studied the effect of dimples and bumps on the performance of a NACA 4415 airfoil. They found that the separation of flow occurs at $12^{\circ}$ for a smooth airfoil, but occurs at around $16^{\circ}$ for the modified airfoils. They also found that both textured airfoils increased lift when compared to the smooth one for all AOA.

Baweja et al. (2016) aimed to improve the performance of a NACA 22112 airfoil by introducing dimples and bumps of various diameters at $68 \%$ of chord length for varying AOA for a $R e$ of $6 \times 10^{6}$. They also found that while the outward dimples (bumps) increased lift, they also increased drag, which reduced the overall L/D. They found that the inward dimples are more effective in keeping the flow attached to the surface for a reduced drag.

Dhiliban et al. (2013) carried out simulations on a NACA 0018 airfoil for subsonic flow for AOA of -20 to $20^{\circ}$. The roughness is introduced at various locations along the airfoil on both the upper and lower surface. They found that having texture on the bottom surface is more effective in increasing the performance of the airfoil, especially at $90 \%$ of chord length for a positive AOA and $60 \%$ of chord length for negative AOA.

Chear and Dol (2015) studied the influence of dimples on the model of a car. They found that a car model with dimples experiences a turbulent boundary layer accompanied by a delayed flow separation. They also found that $C_{d}$ is reduced by $1.95 \%$ for a Dimple Ratio of 0.4 .

Faruqui et al. (2014) studied the effect of a bumpy surface near the trailing edge of a NACA 4315 airfoil. The bumps were located at $80 \%$ of chord length on the upper surface of the airfoil. The CFD analysis was done for varying AOA for 0 to $20^{\circ}$. They found that a bumpy surface delayed flow separation and increased the stall angle from $9^{\circ}$ for a smooth airfoil to $15^{\circ}$ for the bumpy airfoil.

Kalkur (2017) studied the effect of a triangular vortex generator on the flow behavior around a NACA 4415 airfoil at varying locations along the chord length. A CFD analysis was done using Ansys Fluent for AOA from 0 to $25^{\circ}$. He found that the airfoils 
with vortex generators at 25 and 50\% of chord in fact reduced lift and increased drag when compared to the smooth airfoil. However, the airfoil with the vortex generator at $75 \%$ of chord increased the stall angle by $2^{\circ}$ and increased the $C_{l}$ by as high as $5 \%$ with a drag reduction of $1 \%$ at higher AOA.

Mustak et al. (2017) studied the effect of hexagonal protrusions on the aerodynamic efficiency of a NACA 4415 airfoil. The flow was studied using a wind tunnel for subsonic airspeeds for AOA of 0 to $18^{\circ}$. It was found that for the smooth airfoil, the flow separated at $12^{\circ}$, but for the textured airfoil, it separated at $16^{\circ}$. The airfoil with bumps also shows a $19.3 \%$ increase in lift, a $48.39 \%$ reduction in drag and an overall $53.75 \%$ increase in $\mathrm{L} / \mathrm{D}$ performance.

Srivastav (2012) studied the effect of adding inward and outward dimples to a NACA 0018 airfoil. The lift, drag and flow separation are studied at varying AOA for a Re of 320000. The dimples and bumps are of identical diameter and depth/height and are placed at about $50 \%$ of chord. He found that the dimpled configuration has better performance and also suggested the further research of a smart dimple matrix to reduce flow separation and improve performance.

Reducing pressure drag is of huge interest as it reduces fuel consumption, increases safety, and improves the overall performance of an aircraft. The study of surface features, such as dimples and vortex generators (bumps), is of great interest. As found by Bogdanović-Jovanović et al. (2012), drag reduction in a dimpled sphere can be as high as 30\%. This can add up to significant reduction in energy losses. However, there is still more research required to fully understand the impact of surface features before they can be applied on a large scale. This study aims to further study the effects of surface feature, particularly for a new shape. Computational fluid dynamics analysis for different airfoil models is done to understand fluid flow over the wing and the influence of dimples and bumps over the performance of the airfoil.

\section{METHODOLOGY}

The research was conducted as a design study. The airfoil models were designed using SolidWorks and the CFD analysis was conducted using Ansys Fluent.

For the simulation, the flow conditions were set for the atmospheric conditions at a height of $9000 \mathrm{~m}$ with an ambient air temperature of $229.73 \mathrm{~K}$, an air pressure of $30.82 \mathrm{kPa}$, a freestream density of $0.4671 \mathrm{~kg} \cdot \mathrm{m}^{-3}$ and a dynamic viscosity of $1.493 \times 10^{-5} \mathrm{~N} \cdot \mathrm{m}^{-2} \cdot \mathrm{s}$. The simulation was conducted for subsonic flow conditions at Mach 0.6. The speed of sound for the flow conditions was $303.8 \mathrm{~m} \cdot \mathrm{s}^{-1}$ and the resulting freestream velocity was $182.28 \mathrm{~m} \cdot \mathrm{s}^{-1}$. The Reynold's number for the simulation was 1425703.081. The simulation used the Shear Stress Transport $k$ - $\omega$ turbulence model, a Reynolds-Averaged Navier-Stokes (RANS) model, owing to its ability to account for the transport of the principal shear stress in adverse pressure gradient boundary layers near the wall while simultaneously being able to use the $k-\varepsilon$ model in the freestream region.

The surface modifications were done on a straight NACA 0012 airfoil with a chord length of $0.25 \mathrm{~m}$ and a span on $0.25 \mathrm{~m}$. The surface area of the resulting airfoil model is $0.0625 \mathrm{~m}^{2}$. All modified designs were compared to the clean airfoil model in Fig. 1 .

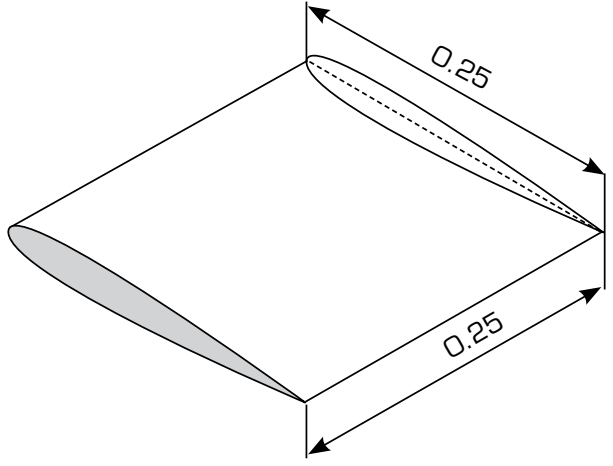

Figure 1. Model of the clean airfoil. 
For designing the airfoil features, the concavity of the feature, i.e., whether it is a dimple or a bump, and the shape of the feature were considered. Therefore, the designs were based on four basic templates: spherical dimples, spherical bumps, pyramidical dimples and pyramidical bumps.

The spherical features were designed to have a diameter of $5 \mathrm{~mm}$ and a depth/height of $2.5 \mathrm{~mm}$. The pyramidical features were designed to have a square base with a side length of $5 \mathrm{~mm}$ and a depth/height of $5 \mathrm{~mm}$.

\section{Design 1}

Design 1 is a dimpled spherical pattern with three rows of dimples at 10, 20 and 30\% of chord (Fig 2).

(a)

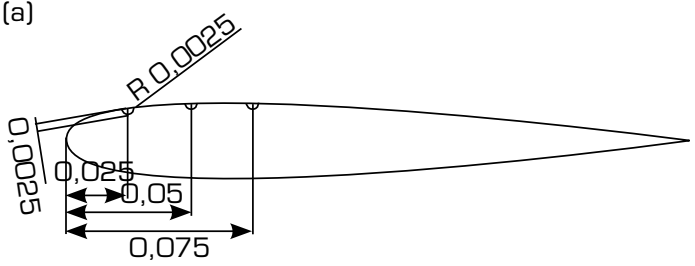

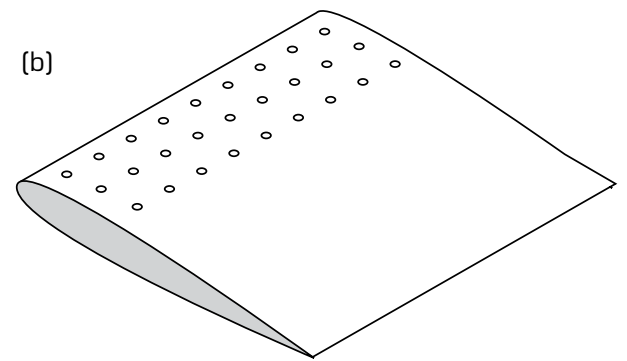

Figure 2. (a) Sketch of design 1; (b) 3D model of design 1.

\section{Design 2}

Design 2 is a "bumped" spherical pattern with three rows of bumps at 10, 20 and 30\% of chord (Fig. 3).
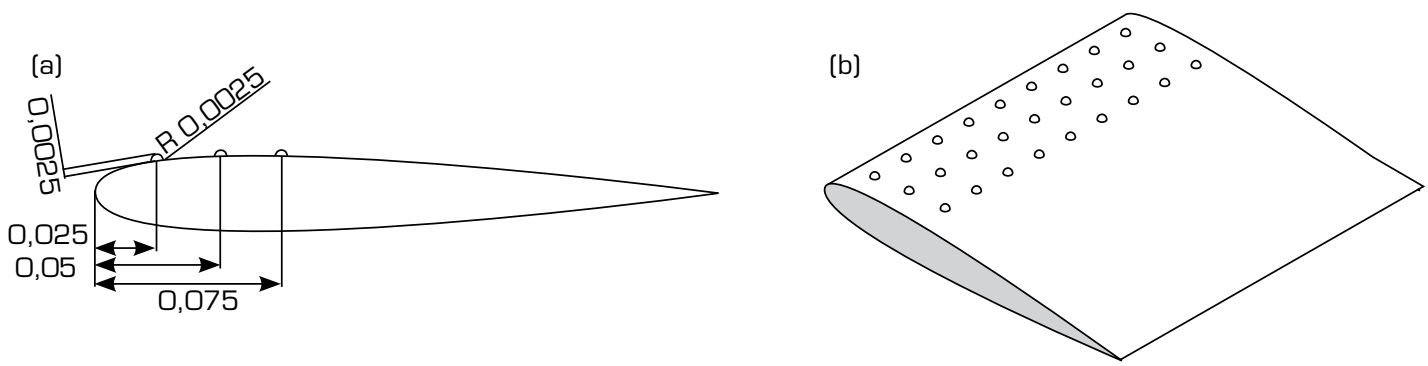

Figure 3. (a) Sketch of design 2; (b) 3D model of design 2.

\section{Design 3}

Design 3 is a compound spherical pattern, which incorporates both dimples and bumps with four rows at 10, 20, 30 and 40\% of chord (Fig. 4). The dimples and bumps were created the same way as they were for designs 1 and 2.

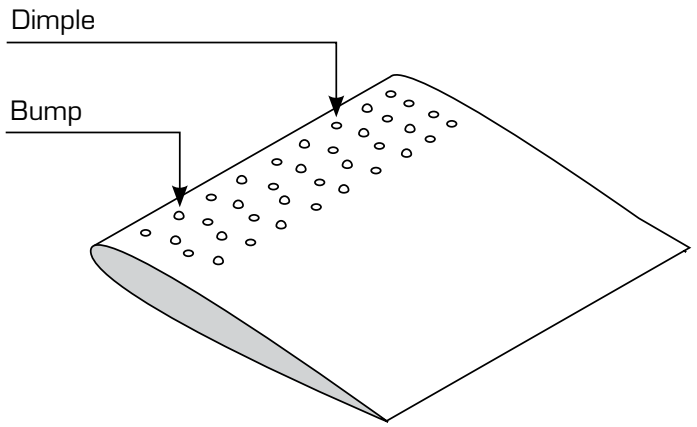

Figure 4. The $3 \mathrm{D}$ model of design 3.

\section{Design 4}

Design 4 is a dimpled pyramidical pattern with three rows of dimples at 10, 20 and 30\% of chord (Fig. 5). 
(a)

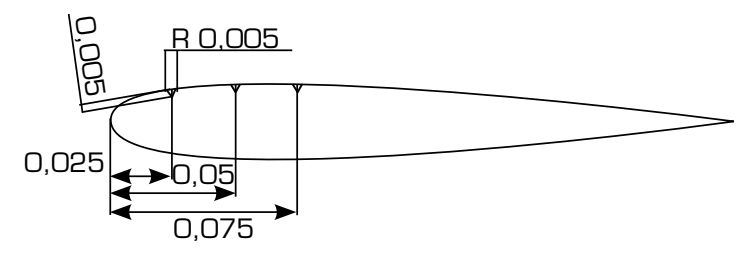

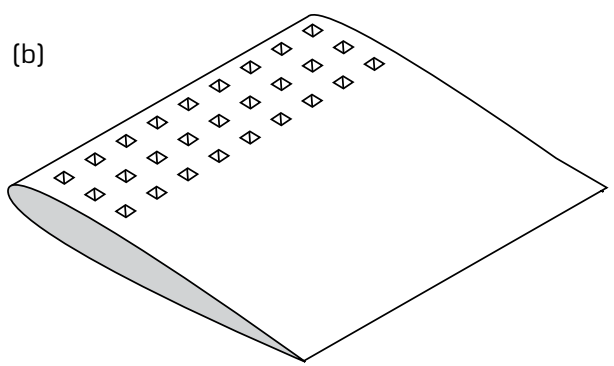

Figure 5. (a) Sketch of design 4; (b) 3D model of design 4.

\section{Design 5}

Design 5 is a "bumped" pyramidical pattern with three rows of bumps at 10, 20 and $30 \%$ of chord (Fig. 6).
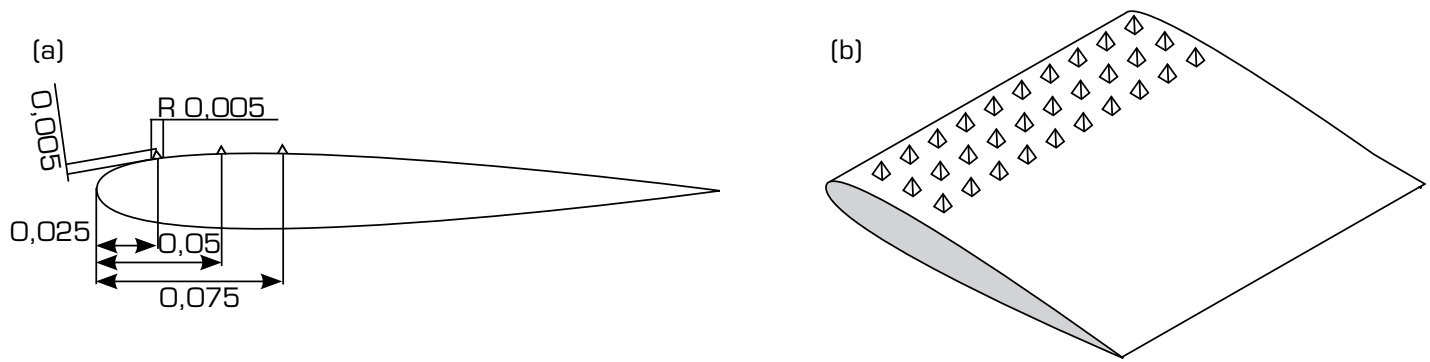

Figure 6. (a) Sketch of design 5; (b) 3D model of design 5.

\section{Design 6}

Design 6 is a compound pyramidical pattern which incorporates both dimples and bumps with four rows at 10, 20, 30 and $40 \%$ of chord (Fig. 7). The dimples and bumps were created the same way as they were for designs 4 and 5.

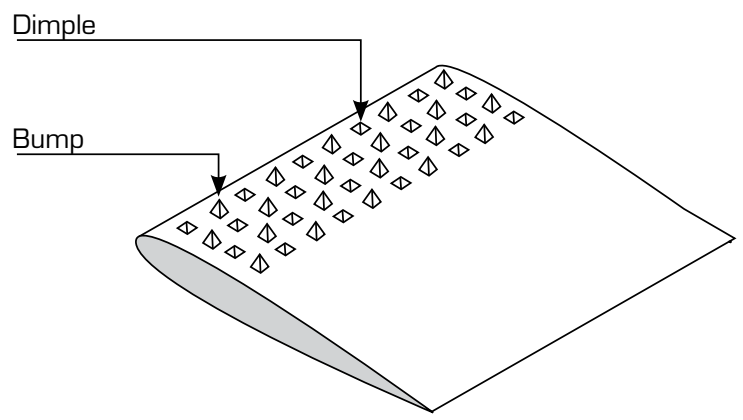

Figure 7. The 3D model of design 6.

The models were simulated using Ansys Fluent. The models were first prepared using SpaceClaim by constructing an enclosure around the airfoil that would be used as the far-field for the external flow. The dimensions of the enclosure are: $\mathrm{x}=3.25 \mathrm{~m}$; $y=3.025 \mathrm{~m} ; \mathrm{z}=1.75 \mathrm{~m}$. The volume of the resulting computational domain is $17.205 \mathrm{~m}^{3}$.

The inlet and outlet of the geometry are defined along with the symmetry. Also, the various parts of the wing are named: the textures, the wing surface and the wing trailing edge. The element size for the wing surface was taken to be about $0.015 \mathrm{~m}$, for the trailing edge it was taken to be $0.00055 \mathrm{~m}$ and for the surface features (dimples and bumps) it was taken to be $0.0007 \mathrm{~m}$.

The first layer thickness, $y$, is determined from the freestream velocity, the freestream density, the dynamic viscosity, the reference length (chord length), and the desired $\mathrm{y}^{+}$value. As the models require enhanced wall treatment, the value of $\mathrm{y}^{+}$is taken to be 1 . Therefore, the first layer thickness is determined to be $y=4.2 \times 10^{-6} \mathrm{~m}$. The models were meshed with an unstructured, tetrahedral mesh with prism boundary layers in the inflation region (Fig. 8). 
(a)

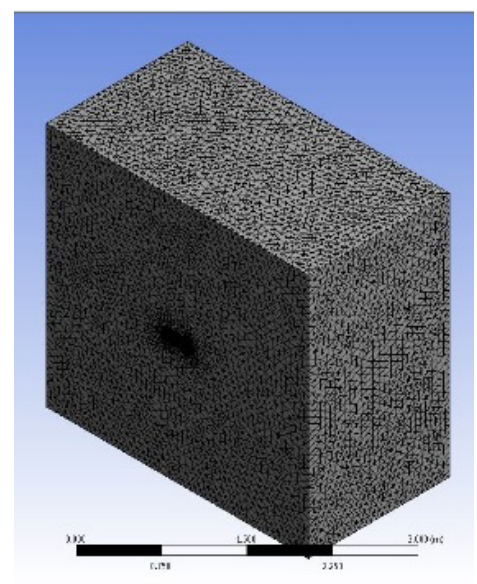

[c]

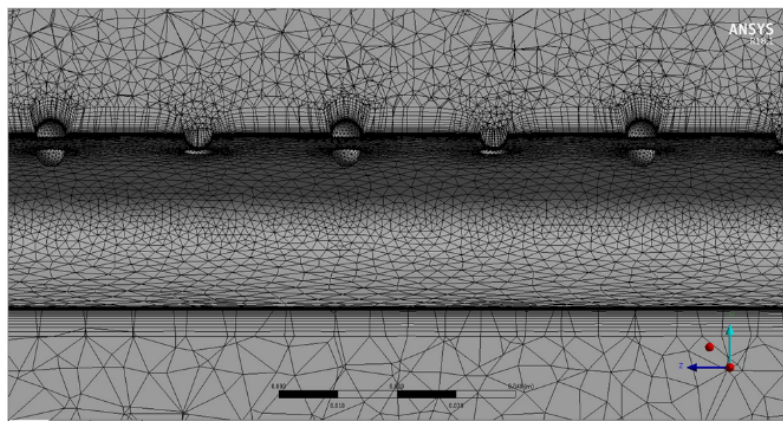

(b)

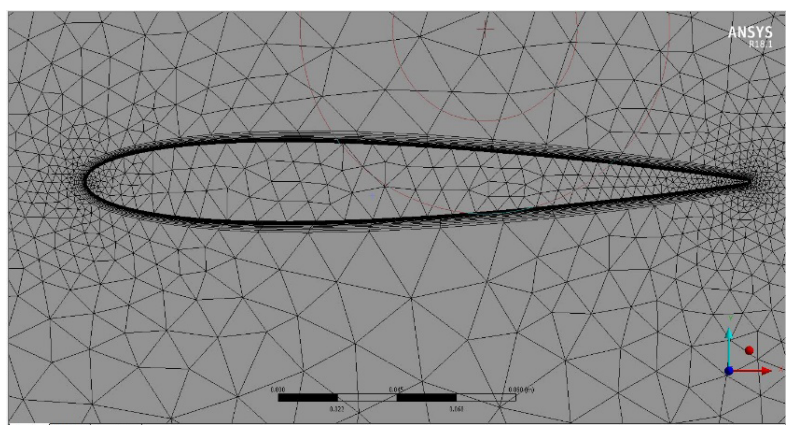

[d]

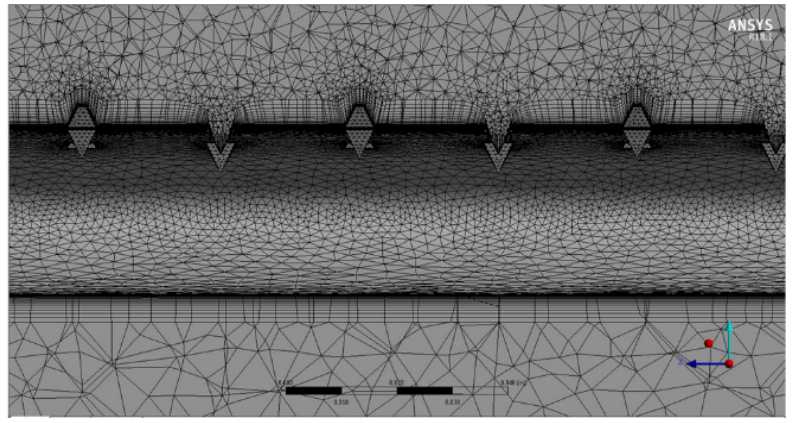

Figure 8. (a) Mesh of entire geometry. (b) Mesh around wing showing the inflation layer. (c) Mesh of spherical features. (d) Mesh of pyramidical features.

The mesh quality is checked using the skewness metric. It is recommended to keep the maximum skewness value below 0.95 .

The maximum skewness values were all well within the acceptable limit (Table 1).

Table 1. Skewness values.

\begin{tabular}{cccc}
\hline Design & Max. skewness & Min. skewness & Average skewness \\
\hline Clean & 0.87703 & $3.94461 \times 10^{-6}$ & 0.33712 \\
\hline Design 1 & 0.87251 & $6.18718 \times 10^{-6}$ & 0.31084 \\
\hline Design 2 & 0.89936 & $2.09600 \times 10^{-6}$ & 0.31087 \\
\hline Design 3 & 0.89707 & $2.60595 \times 10^{-6}$ & 0.30711 \\
\hline Design 4 & 0.89098 & $6.05100 \times 10^{-6}$ & 0.30854 \\
\hline Design 5 & 0.89655 & $7.52169 \times 10^{-6}$ & 0.31188 \\
\hline Design 6 & 0.89098 & $6.05100 \times 10^{-6}$ & 0.30854 \\
\hline
\end{tabular}

Table 2 shows the number of elements and nodes for each model. 
Table 2. Number of elements and nodes for each of the models.

\begin{tabular}{ccc}
\hline Design & Number of nodes & Number of elements \\
\hline Clean & 313857 & 911070 \\
\hline Design 1 & 689053 & 1753376 \\
\hline Design 2 & 786412 & 1966856 \\
\hline Design 3 & 788313 & 1977832 \\
\hline Design 4 & 594144 & 1538337 \\
\hline Design 5 & 676955 & 1418948 \\
\hline Design 6 & 785382 & 1980628 \\
\hline
\end{tabular}

For the simulation, the operating pressure was set to $0 \mathrm{~Pa}$ as the flow is external. Since the flow is subsonic and the behavior is to be observed after the flow profile has formed, the pressure-based and steady state solvers are used. The fluid is set to have ideal gas density and Sutherland viscosity. The boundary conditions for the model are defined in Table 3.

Table 3. Boundary conditions.

\begin{tabular}{cc}
\hline Boundary & Type \\
\hline Symmetry & Symmetry \\
\hline Interior_enclosure_enclosure & Interior \\
\hline Inlet_pressure_farfield & Pressure-far-field \\
\hline Outlet_pressure & Pressure-outlet \\
\hline Wing & Wall \\
\hline Wing_te & Wall \\
\hline Wing_tip & Wall \\
\hline Dimples_bumps & Wall
\end{tabular}

For the solution methods, the scheme was set to "Coupled", which couples the mass and momentum equations. The spatial discretization settings used are shown in Table 4.

Table 4. Spatial discretization settings.

\begin{tabular}{cc}
\hline Gradient & Least squares cell based \\
\hline Pressure & Second order upwind \\
\hline Density & Second order upwind \\
\hline Momentum & First order upwind \\
\hline Turbulent kinetic energy & First order upwind \\
\hline Specific dissipation rate & First order upwind \\
\hline Energy & First order upwind \\
\hline
\end{tabular}

It should be noted that, after the first convergence, the momentum, turbulent kinetic energy, specific dissipation rate and energy settings are changed to second order upwind.

The cells are initialized using standard initialization where the initial values were the inlet values. The models were simulated for varying AOA: 0, 5, 10 and $15^{\circ}$. The models are run until the residuals converge.

\section{RESULTS AND DISCUSSION}

From post-CFD, the velocity contours and velocity streamlines are generated. For the clean, design 1, design 2 , design 4 and design 5 models, the contours and streamlines are captured on a plane located at $0.125 \mathrm{~m}$ along the $z$-coordinate. For designs 3 
and 6 models, since there is variation in the design along both the $x$ - and $z$-coordinates, two planes are used to capture the two sequences; one at $0.125 \mathrm{~m}$ and one at $0.15 \mathrm{~m}$ (Figs. 9 and 10).

(a)

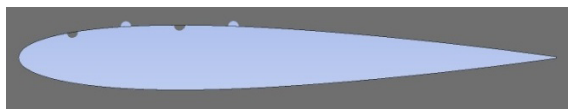

(b)

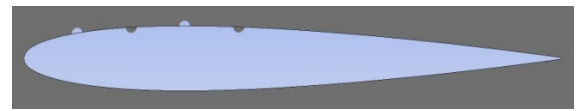

Figure 9. (a) Plane at $0.125 \mathrm{~m}$ for design 3. (b) Plane at $0.15 \mathrm{~m}$ for design 3.

(a)

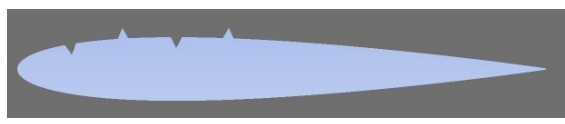

(b)

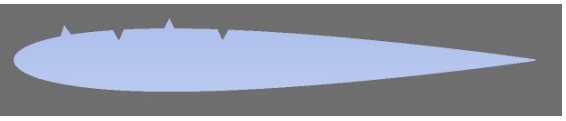

Figure 10. (a) Plane at $0.125 \mathrm{~m}$ for design 6. (b) Plane at $0.15 \mathrm{~m}$ for design 6.

\section{Velocity contours}

The velocity contours for all models are nearly identical at AOA 0, 5 and $10^{\circ}$ (Figs. 11-19). This suggests that the features do not contribute much to the flow behavior at lower AOA.

From the contours of the models with dimples (Figs. 12, 14-16, 18 and 19), the dark blue region inside the dimple suggests a laminar separation bubble (LSB) or a region with vortex formation. These tiny vortices inside the dimples provide energy to the flow and prevent boundary layer separation. Similarly, for the models with bumps (Figs. 13-15, 17-19), there is a dark blue region behind the bumps, which indicates the presence of an LSB. Also, there is a small red region directly above the bumps (Figs. 13-15, 17-19), which shows increased flow momentum due to these imperfections. However, it should also be noted that the red region is strongest for the first bump and gets weaker for each subsequent bump.

(a)

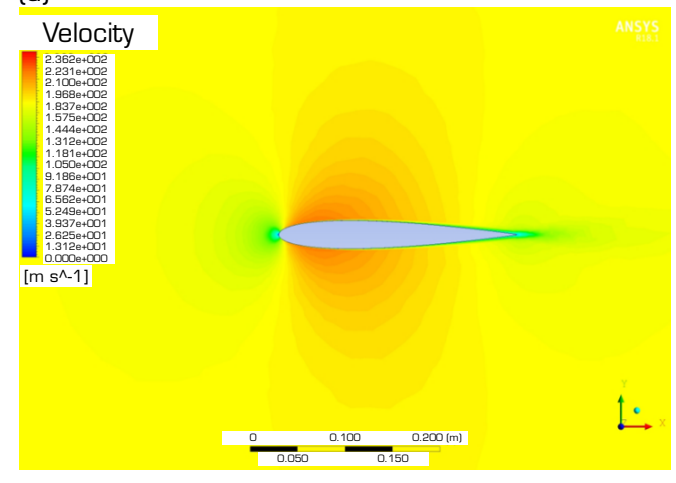

(c)

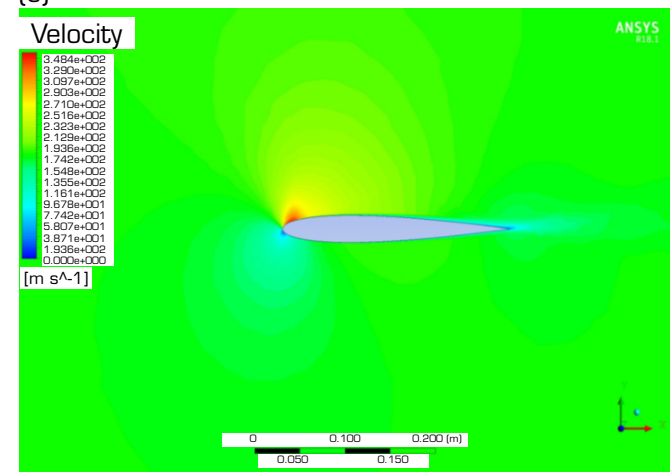

(b)

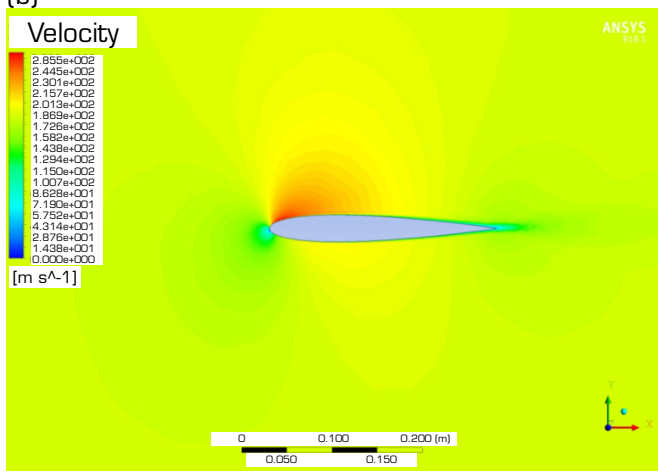

(d)

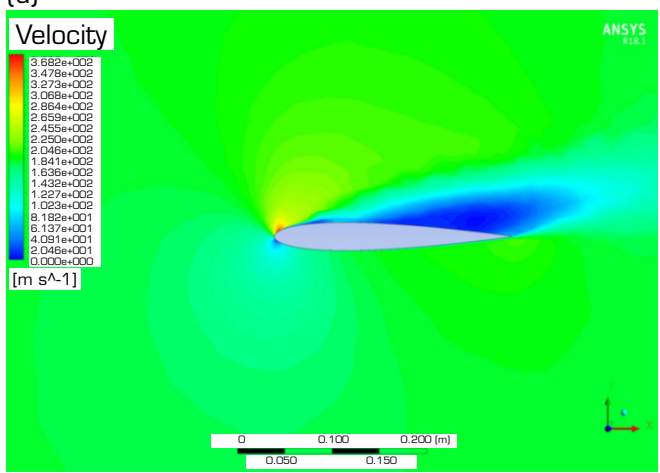

Figure 11. Velocity contours for clean airfoil at (a) $0^{\circ}$, (b) $5^{\circ}$, (c) $10^{\circ}$ and (d) $15^{\circ}$. 
(a)

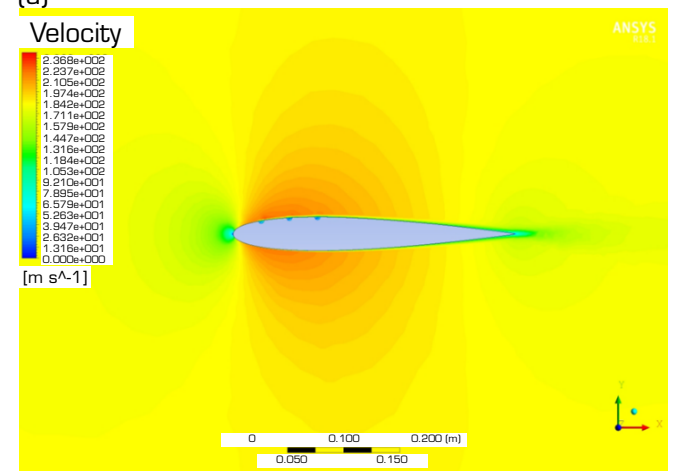

(c)

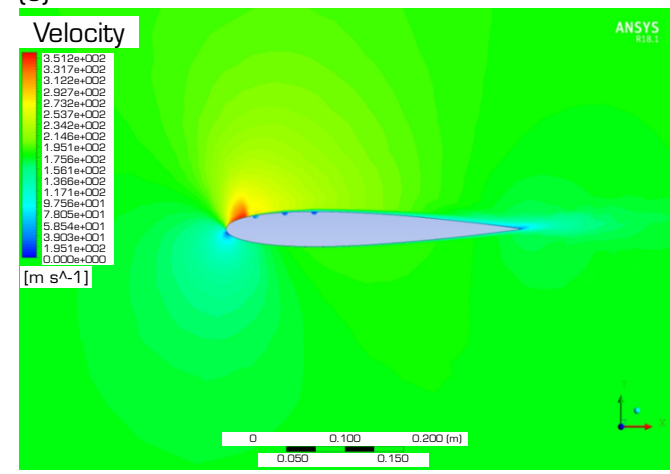

(b)
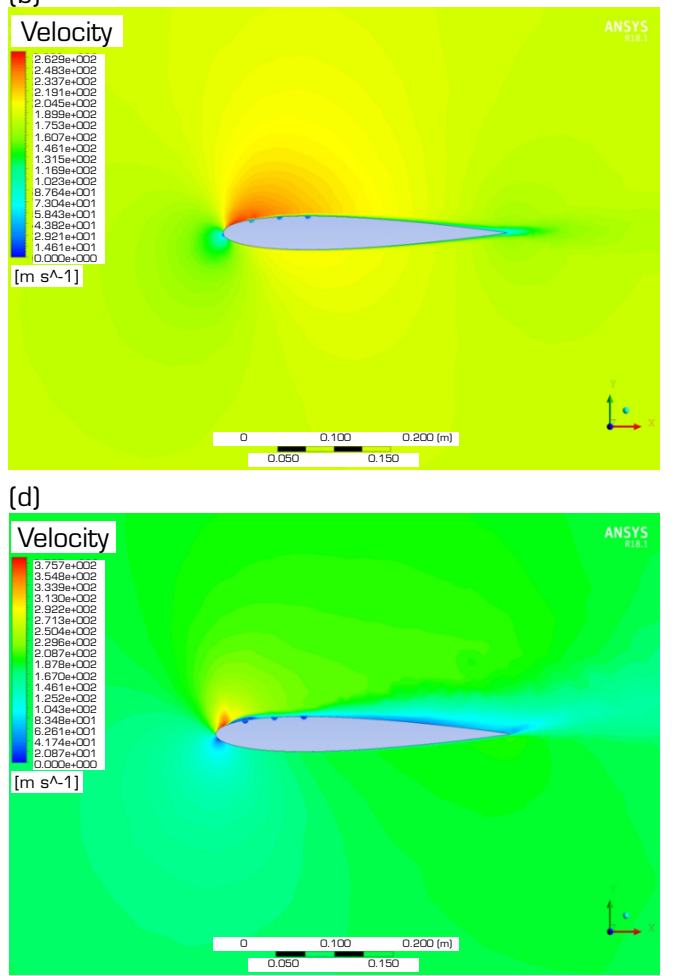

Figure 12. Velocity contours for design 1 at (a) $0^{\circ}$, (b) $5^{\circ}$, (c) $10^{\circ}$ and (d) $15^{\circ}$.

(a)

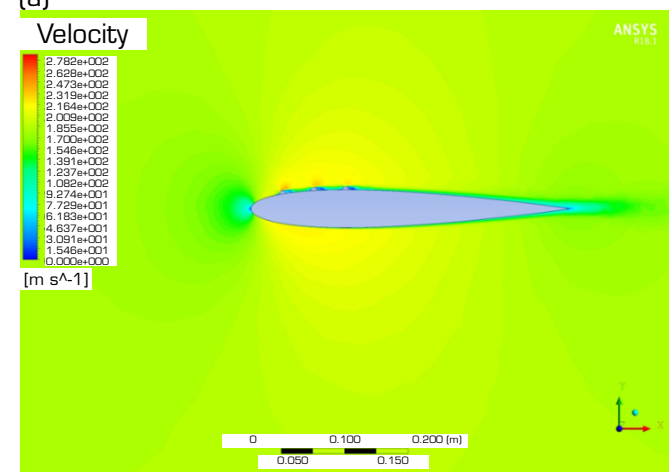

(c)

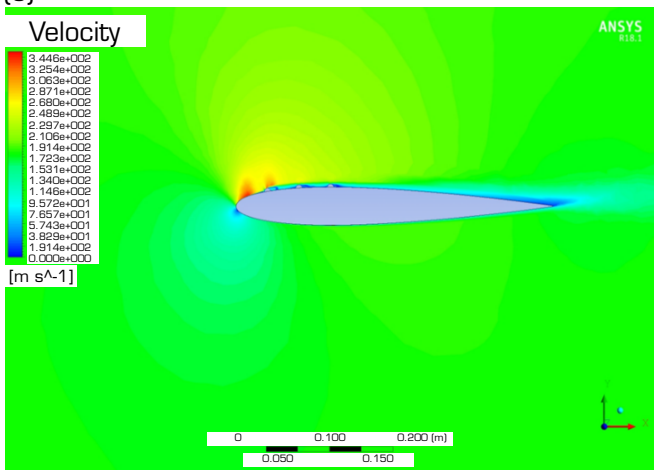

(b)

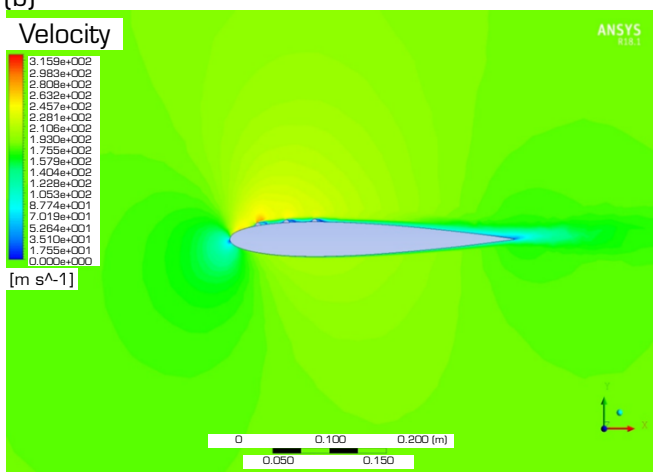

(d)

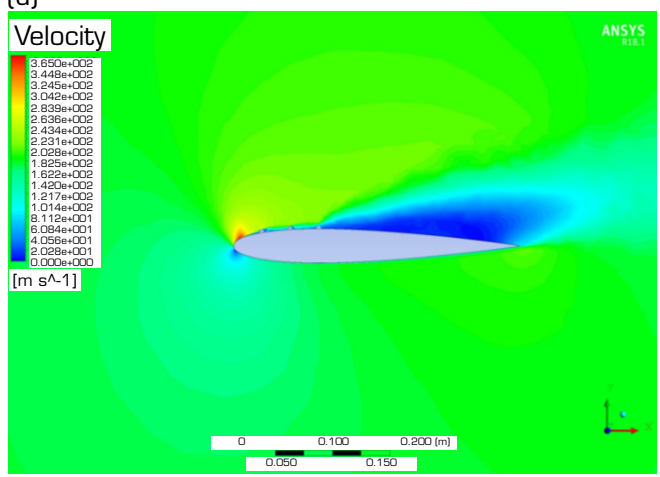

Figure 13. Velocity contours for design 2 at (a) $0^{\circ}$, (b) $5^{\circ}$, (c) $10^{\circ}$ and (d) $15^{\circ}$. 
(a)

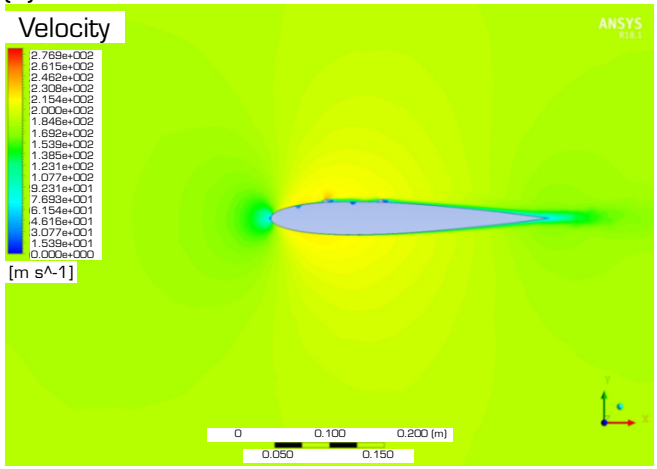

(c)

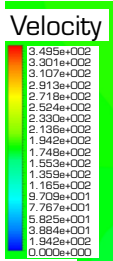

$\left[\mathrm{m} \mathrm{s} \mathrm{s}^{\wedge}-1\right]$

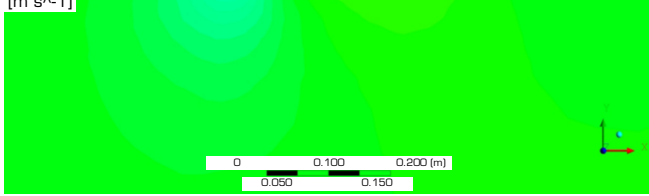

(b)

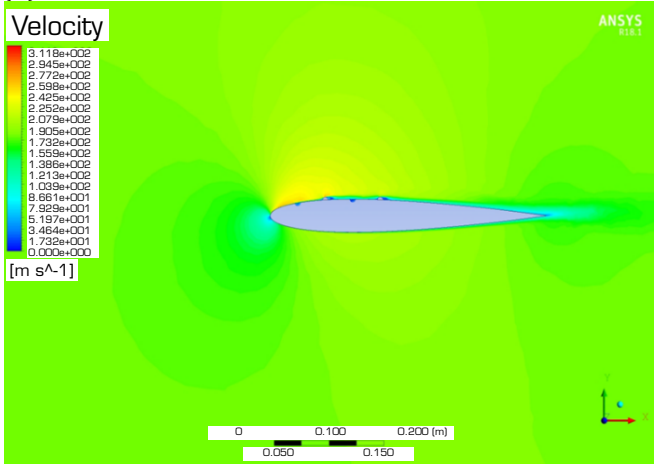

(d)

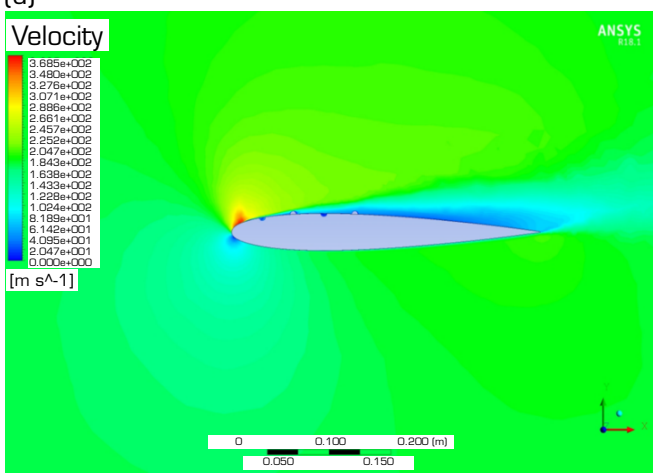

Figure 14. Velocity contours for design 3 for the plane at $0.125 \mathrm{~m}$ at (a) $0^{\circ}$, (b) $5^{\circ}$, (c) $10^{\circ}$ and (d) $15^{\circ}$.

(a)

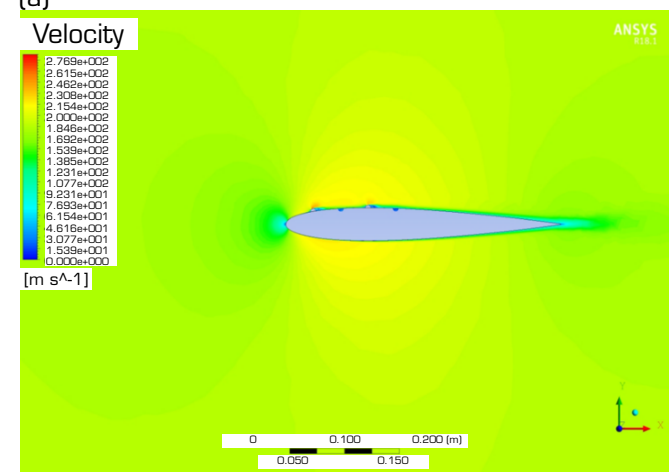

(c)

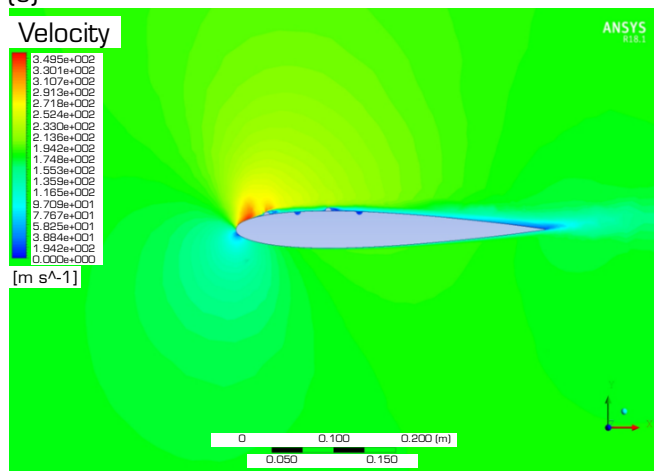

(b)

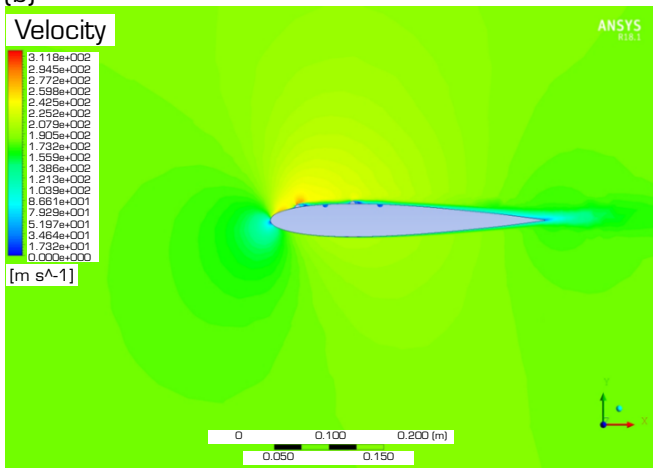

(d)

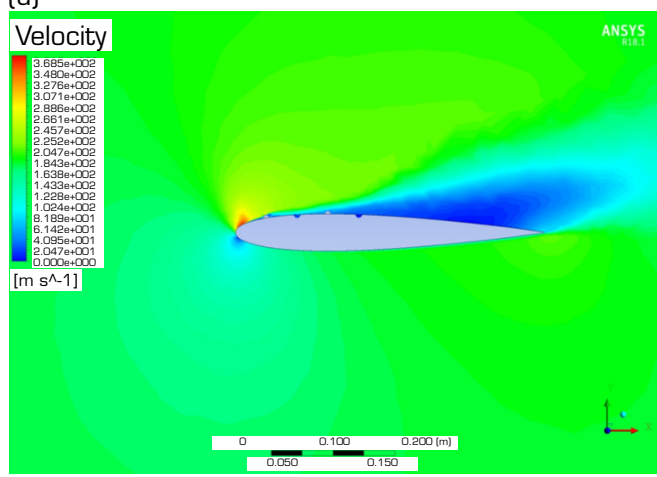

Figure 15. Velocity contours for design 3 for the plane at $0.15 \mathrm{~m}$ at (a) $0^{\circ}$, (b) $5^{\circ}$, (c) $10^{\circ}$ and (d) $15^{\circ}$. 
(a)

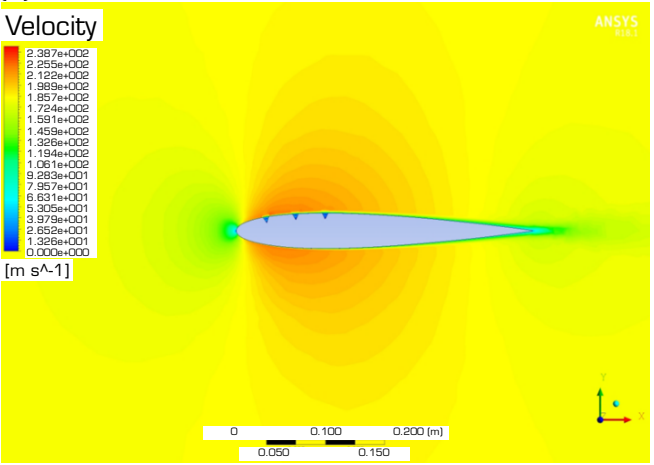

(c)

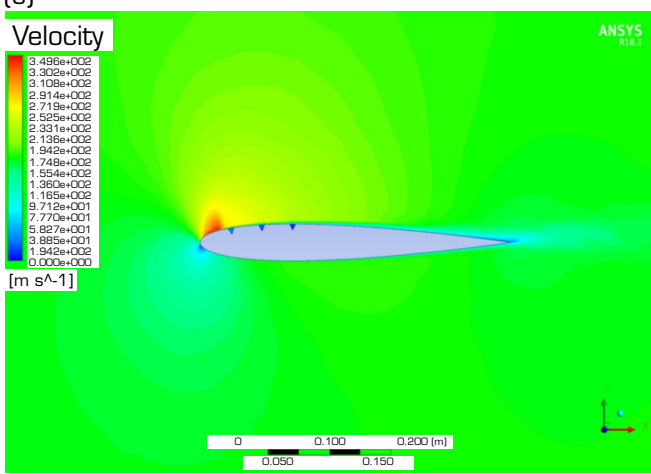

(b)

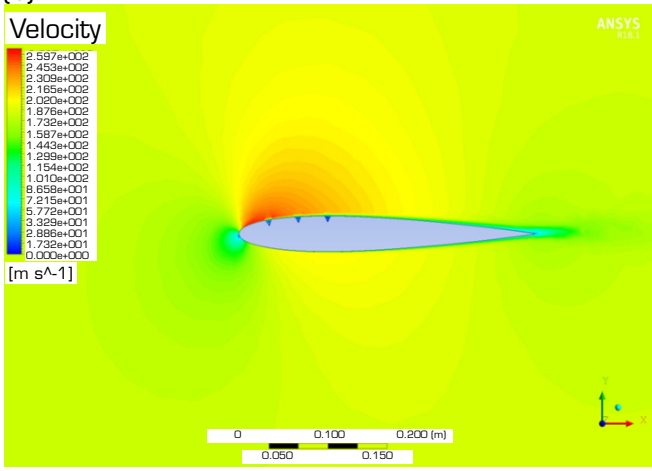

(d)

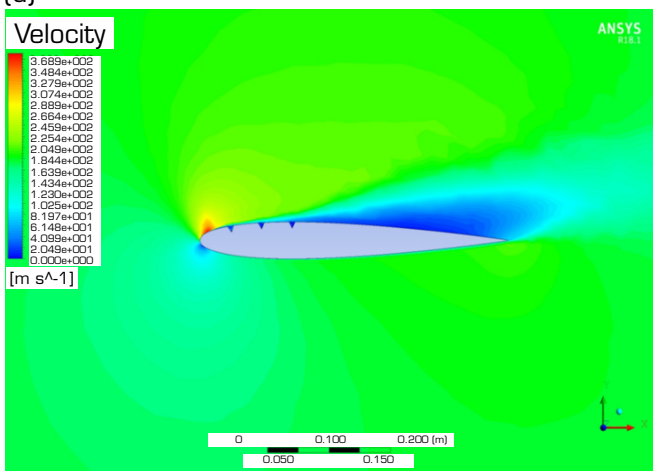

Figure 16. Velocity contours for design 4 at (a) $0^{\circ}$, (b) $5^{\circ}$, (c) $10^{\circ}$ and (d) $15^{\circ}$.

(a)

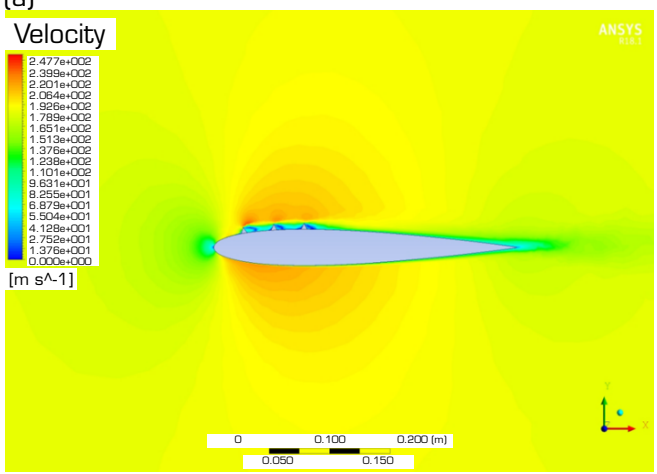

(c)

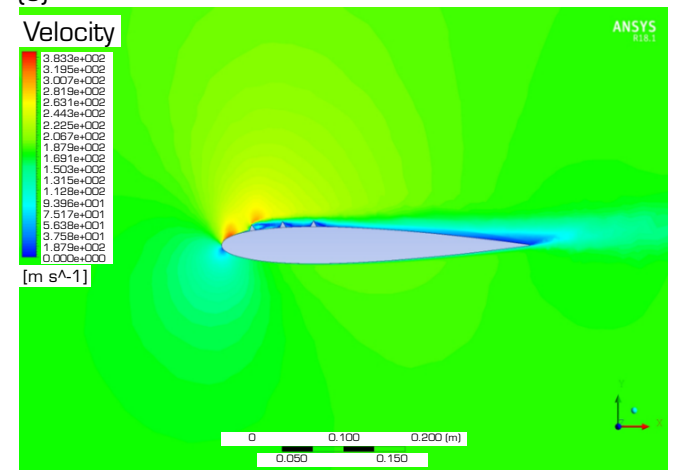

(b)

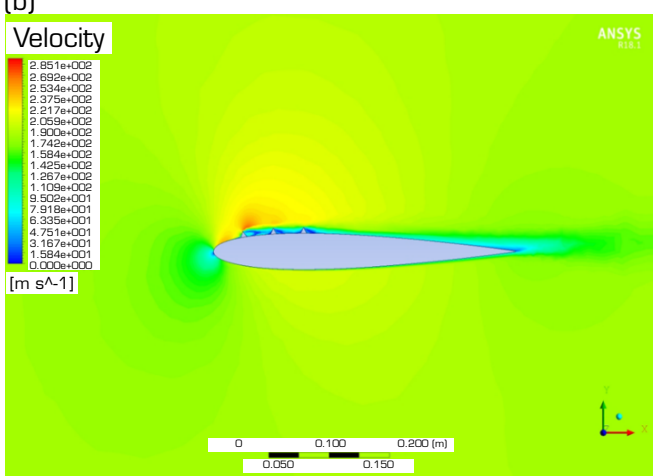

(d)

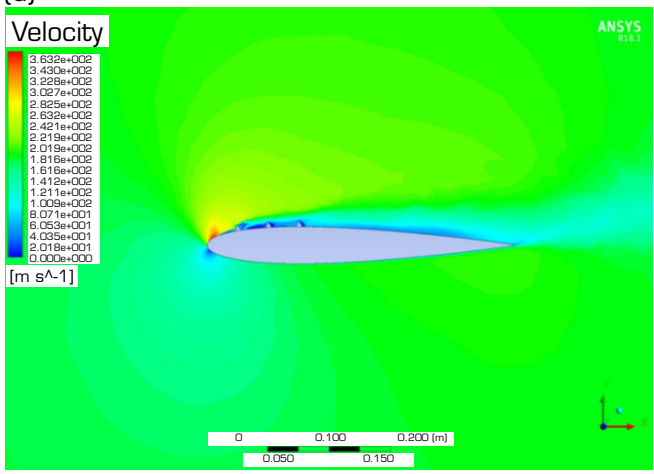

Figure 17. Velocity contours for design 5 at (a) $0^{\circ}$, (b) $5^{\circ}$, (c) $10^{\circ}$ and (d) $15^{\circ}$. 
(a)

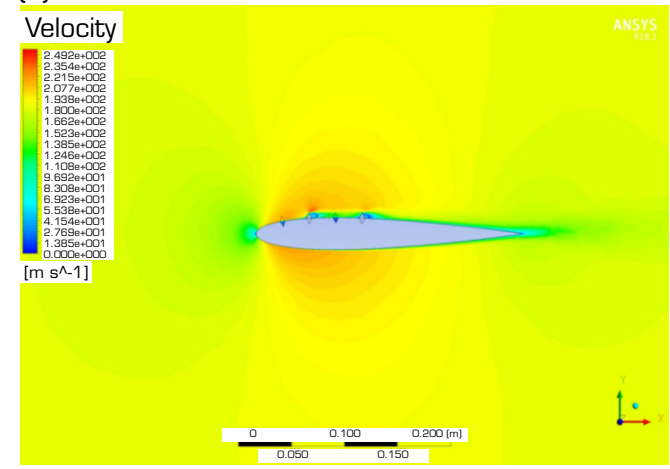

(c)
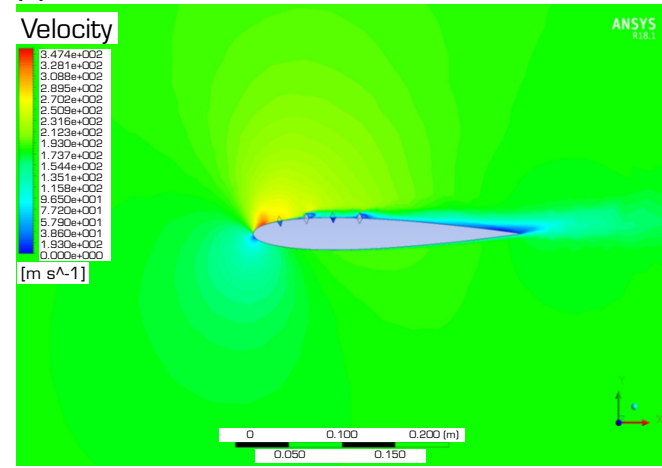

(b)
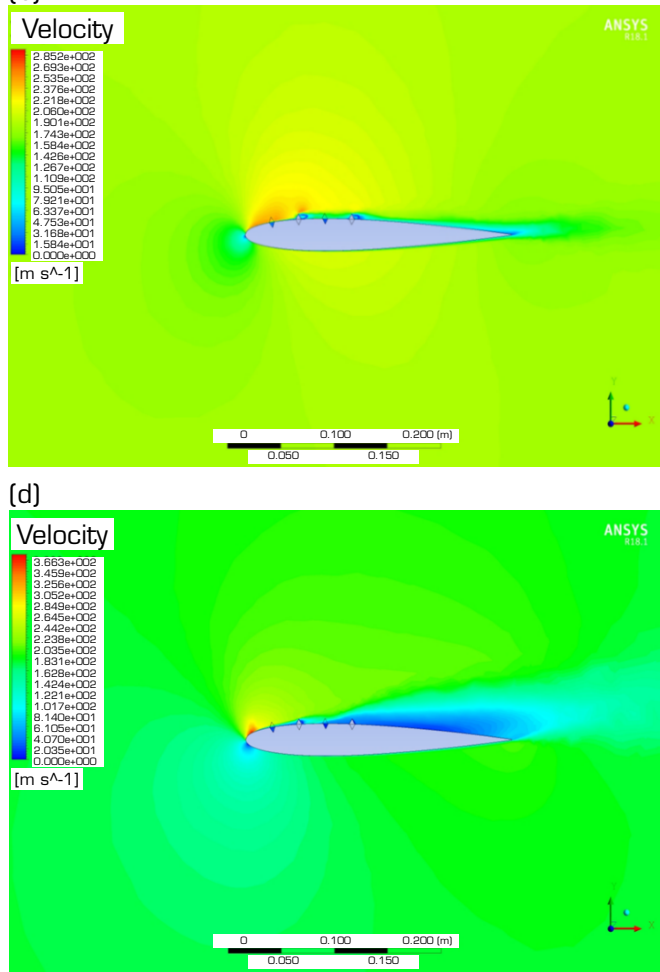

Figure 18. Velocity contours for design 6 for the plane at $0.125 \mathrm{~m}$ at (a) $0^{\circ}$, (b) $5^{\circ}$, (c) $10^{\circ}$ and (d) $15^{\circ}$.

(a)

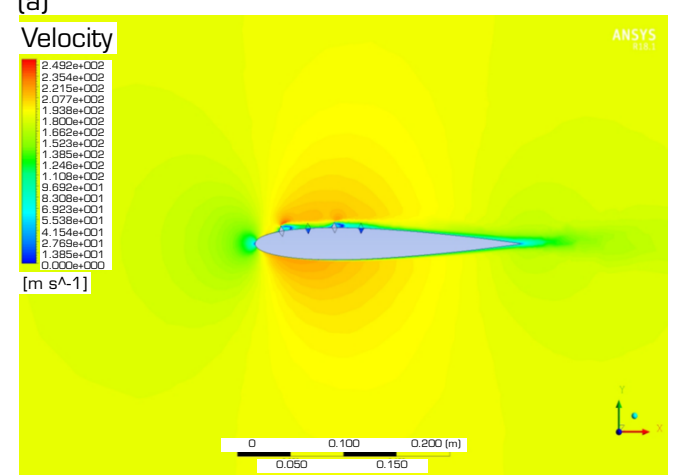

(c)

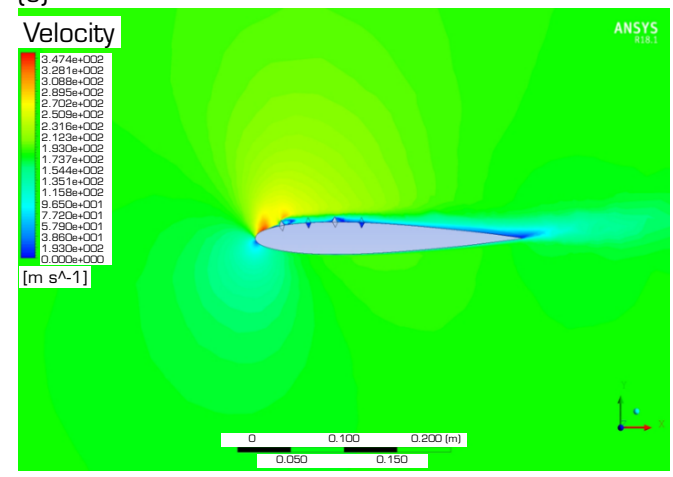

(b)

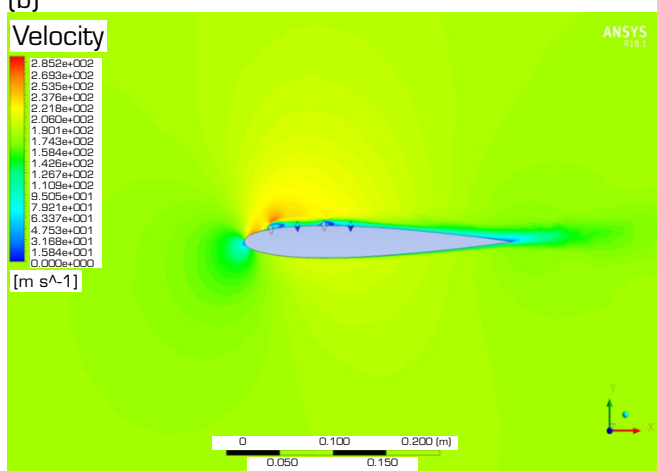

(d)

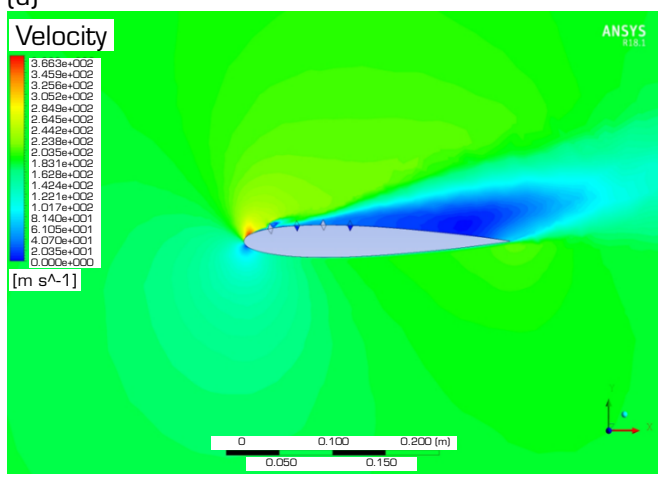

Figure 19. Velocity contours for design 6 for the plane at $0.15 \mathrm{~m}$ at (a) $0^{\circ}$, (b) $5^{\circ}$, (c) $10^{\circ}$ and (d) $15^{\circ}$. 
From the velocity contours, the dark blue region near the trailing edge of many of the $15^{\circ}$ contours, as shown in Figs. 11d, $13 \mathrm{~d}, 15 \mathrm{~d}, 16 \mathrm{~d}$ and $19 \mathrm{~d}$, show a wake region. A wake region indicates that flow is not perfectly aligned with the wing surface and is starting to lose energy; it is essentially disturbed flow.

From the velocity contours, for design 1 (Fig. 12d), design 3 at $0.125 \mathrm{~m}$ (Fig. 14d), design 5 (Fig. 17d) and design 6 at $0.125 \mathrm{~m}$ (Fig. 18d), at $15^{\circ}$, the wake generated is the smallest. For the others, there definitely is disturbance. However, the type of disturbance, whether it is simply low energy flow or recirculating flow, can only be determined by analyzing the streamlines.

\section{Velocity streamlines}

The velocity streamlines show the behavior of the flow particles as they move over the wing. Streamlines can help visualize flow phenomenon, such as flow separation and flow recirculation.

As with the velocity contours, the streamlines are almost identical for 0,5 and $10^{\circ} \mathrm{AOA}$ for all models. The streamlines show that the flow recirculates for the clean model at $15^{\circ}$ (Fig. 20d). All the other models do not experience recirculation of flow, although, they all experience a reduction in flow energy indicated by the blue coloring of the lines towards the trailing edge. Therefore, there is a definite improvement in the airfoil performance and a delay in the boundary layer separation.
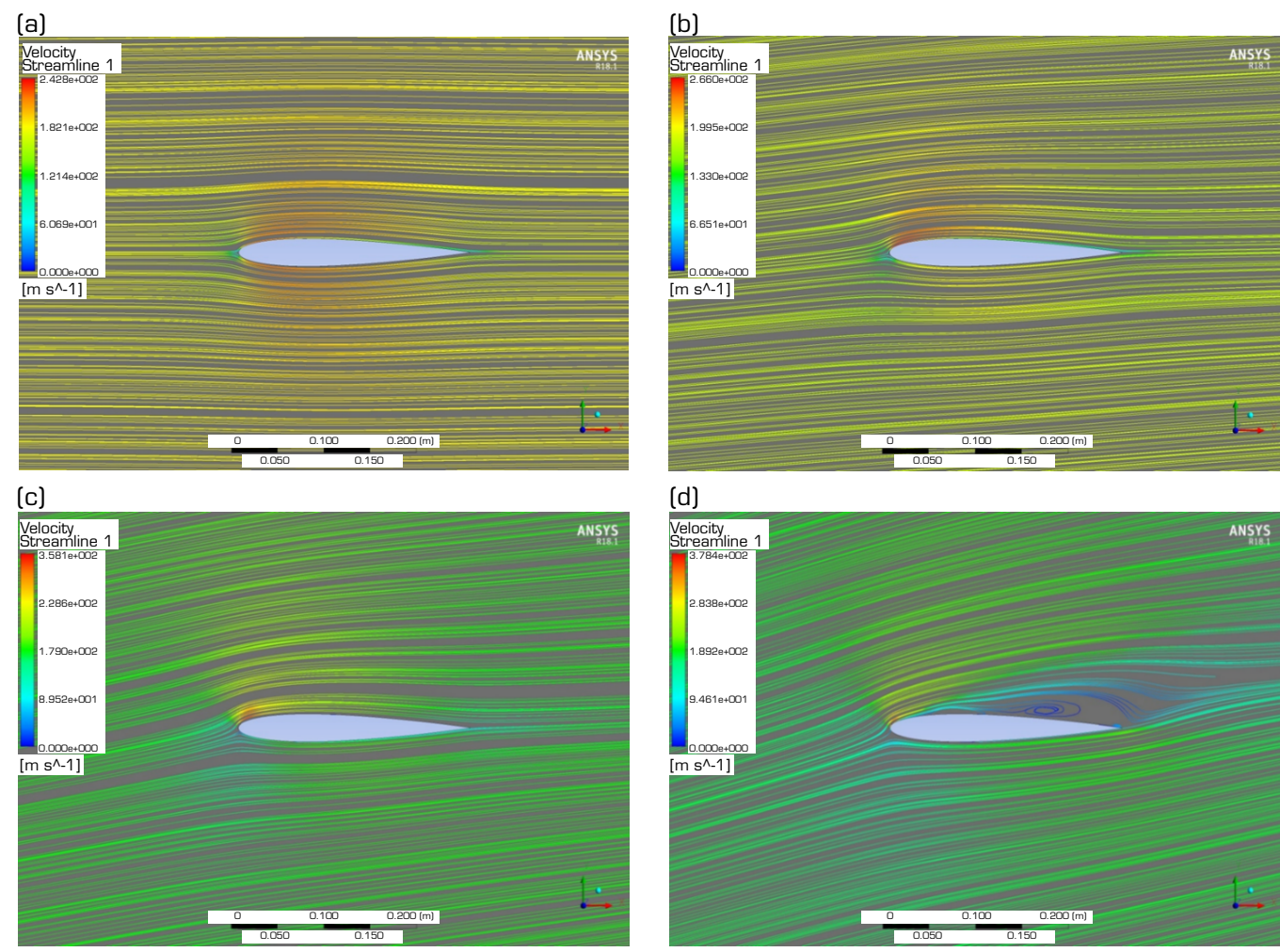

Figure 20. Streamlines for clean airfoil at (a) $0^{\circ}$, (b) $5^{\circ}$, (c) $10^{\circ}$ and (d) $15^{\circ}$.

The flow is most streamlined for design 1 (Fig. 21d) and design 5 (Fig. 26d). Design 3 at 0.125 m (Fig. 23d) and design 6 at $0.125 \mathrm{~m}$ (Fig. 27d) experience a small disturbance where the flow slightly separates from the surface.

Design 3 at $0.15 \mathrm{~m}$ (Fig. 24d) and design 4 (Fig. 25d) experience more of a disturbance as more streamlines are disturbed when compared to design 3 at $0.125 \mathrm{~m}$ (Fig. 23d) and design 6 at $0.125 \mathrm{~m}$ (Fig. 27d).

Design 6 at $0.15 \mathrm{~m}$ (Fig. 28d) has a tiny region of vortex formation directly behind the $2^{\text {nd }}$ bump. This region of separation is an LSB, where the flow separates and generates a local vortex for a small surface area of the wing. This vortex is likely formed due to the bump, as it forms right behind it. This bubble likely provides the flow with extra energy and momentum as the flow starts to separate, seen by the loss of alignment of the streamlines with the surface, but then reattaches right before it leaves the surface at the tip of the trailing edge. This vortex formation was not observed for any spherical bumps, suggesting that pyramidical bumps are better at generating local vortices. 
For design 2 (Fig. 22d), towards the tip of the trailing edge, it can be seen that flow is beginning to recirculate. This flow separation is also delayed when compared with the clean airfoil (Fig. 20d).
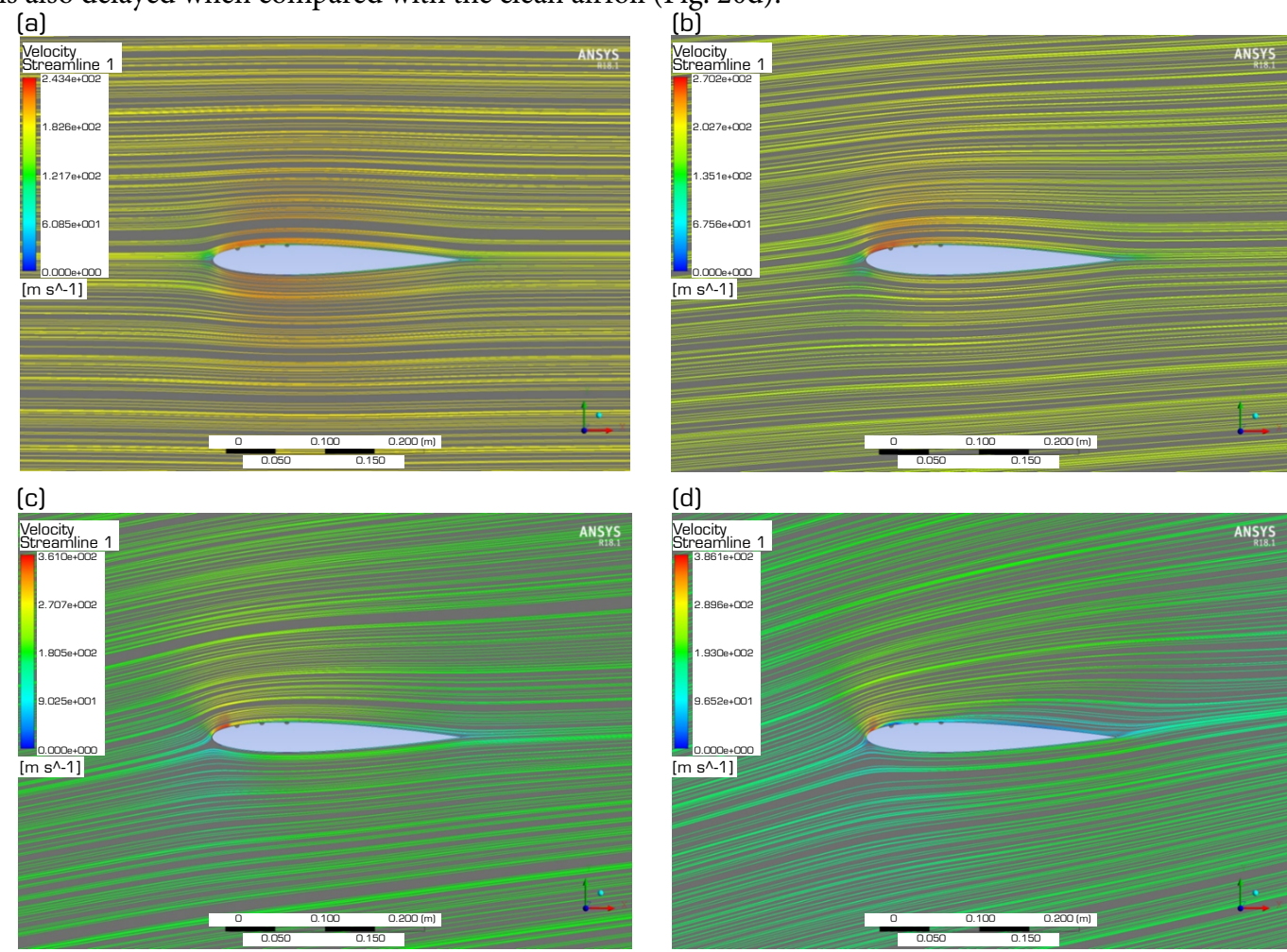

Figure 21. Streamlines for design 1 at (a) $0^{\circ}$, (b) $5^{\circ}$, (c) $10^{\circ}$ and (d) $15^{\circ}$.
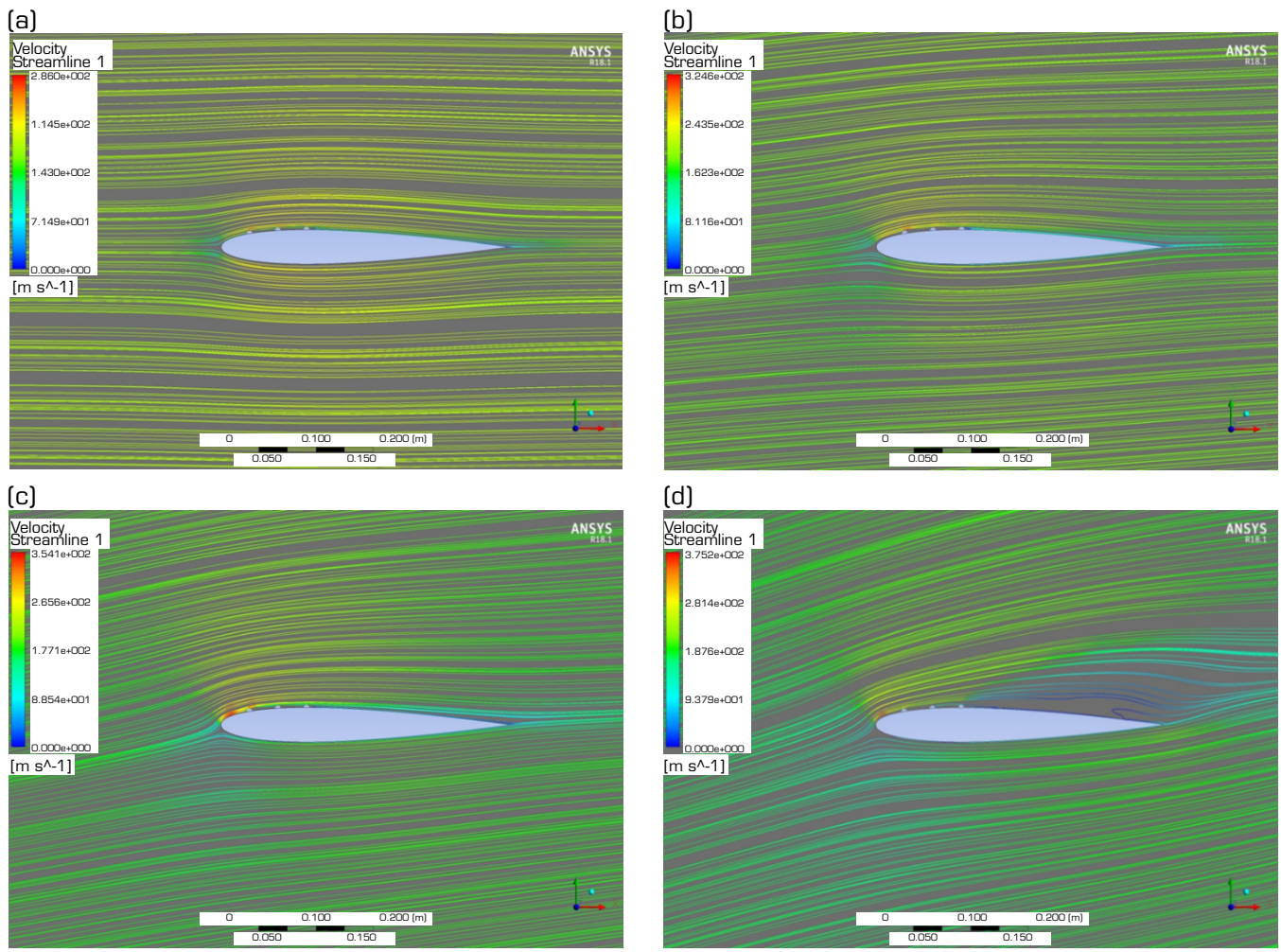

Figure 22. Streamlines for design 2 at (a) $0^{\circ}$, (b) $5^{\circ}$, (c) $10^{\circ}$ and (d) $15^{\circ}$. 
(a)

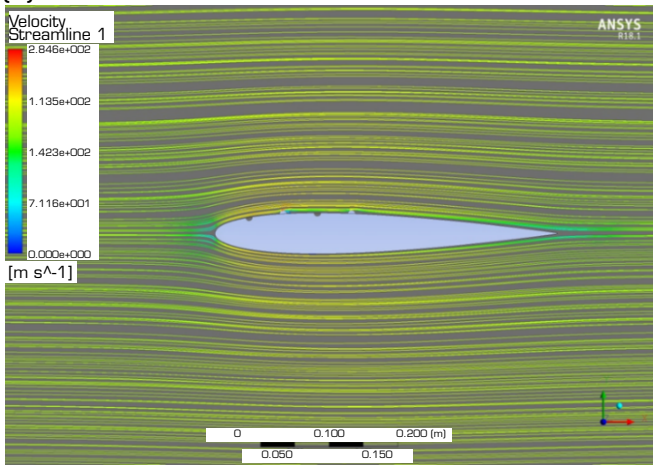

(c)

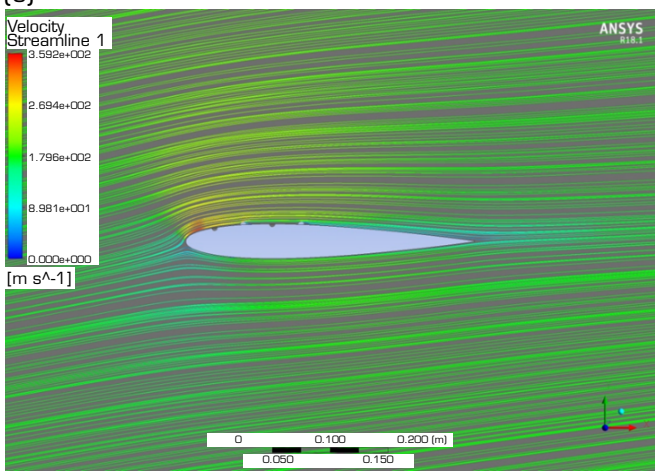

(b)

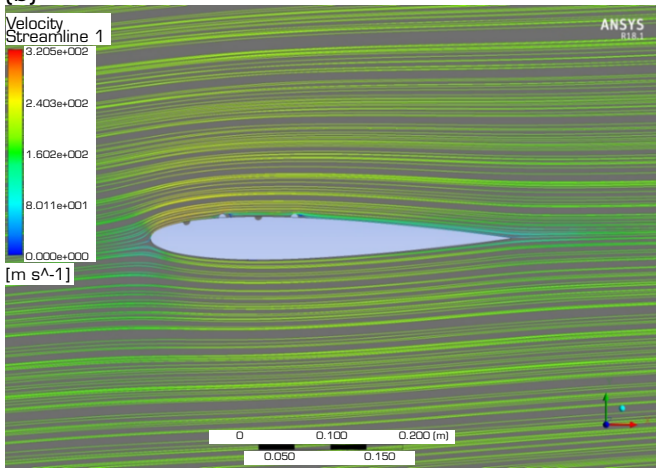

(d)

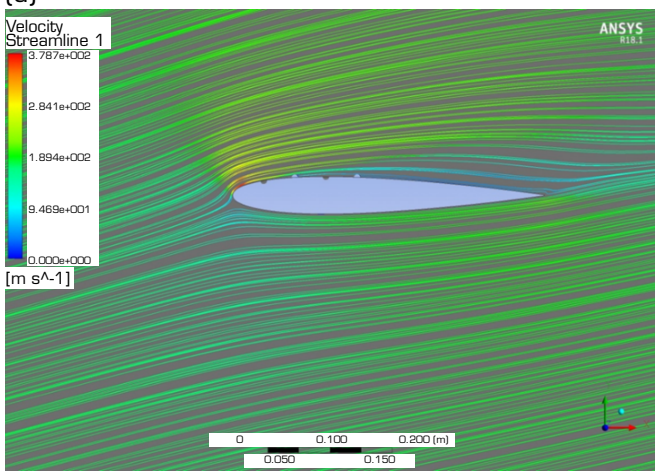

Figure 23. Streamlines for design 3 for the plane at $0.125 \mathrm{~m}$ at (a) $0^{\circ}$, (b) $5^{\circ}$, (c) $10^{\circ}$ and (d) $15^{\circ}$.

(a)

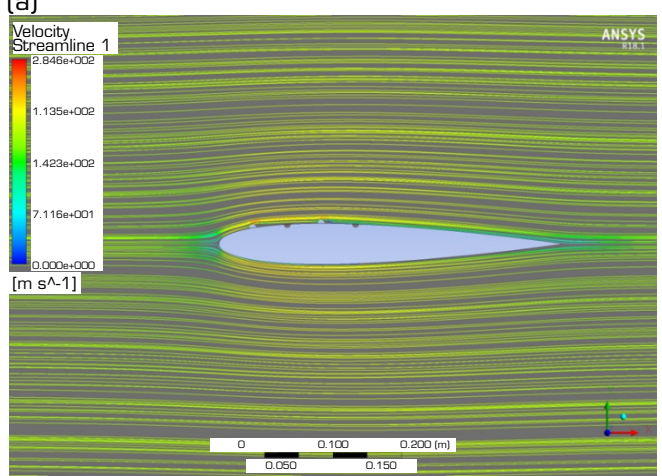

(c)

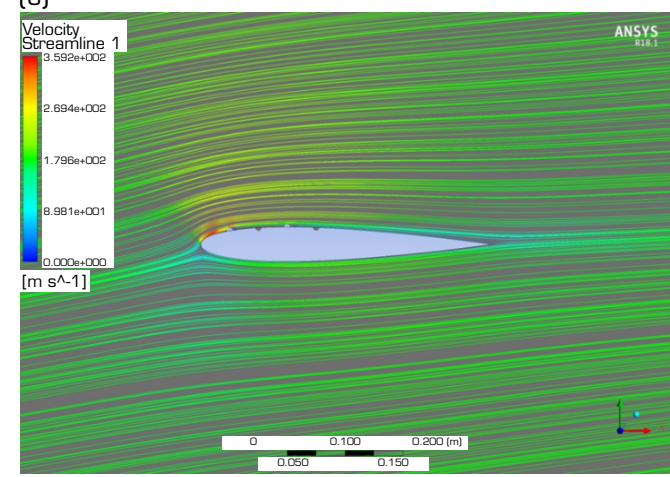

(b)

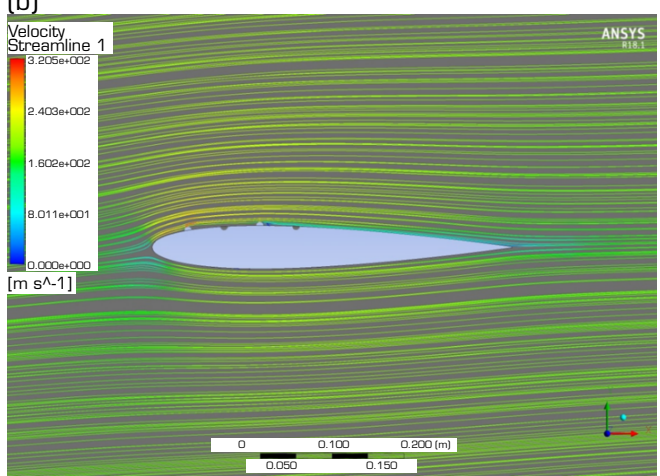

(d)

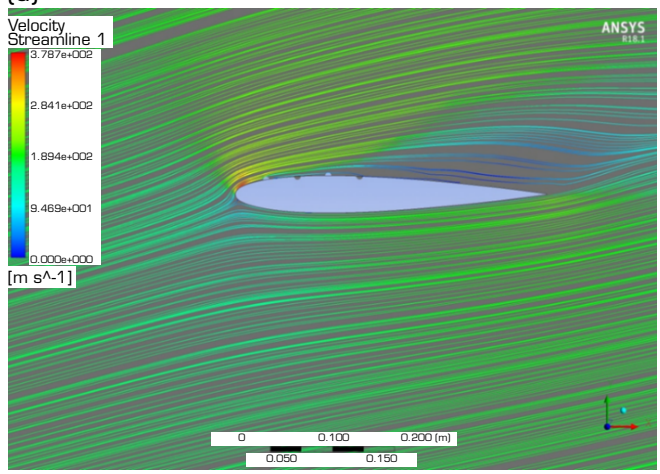

Figure 24. Streamlines for design 3 for the plane at $0.15 \mathrm{~m}$ at (a) $0^{\circ}$, (b) $5^{\circ}$, (c) $10^{\circ}$ and (d) $15^{\circ}$. 
(a)

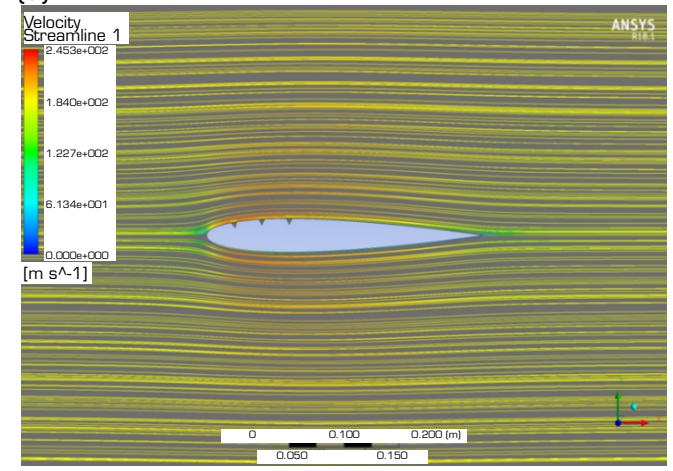

(c)

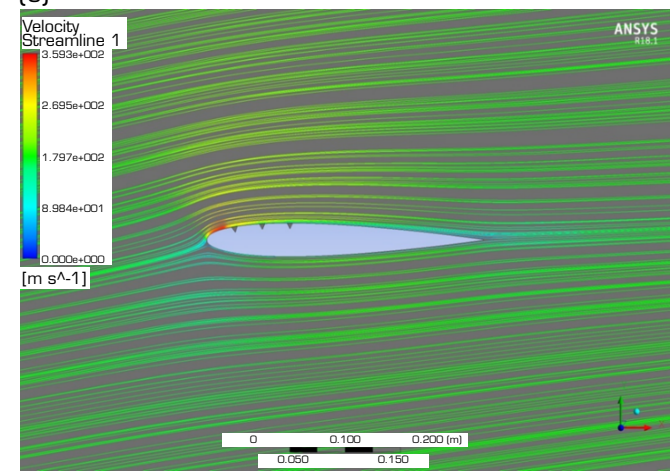

(b)

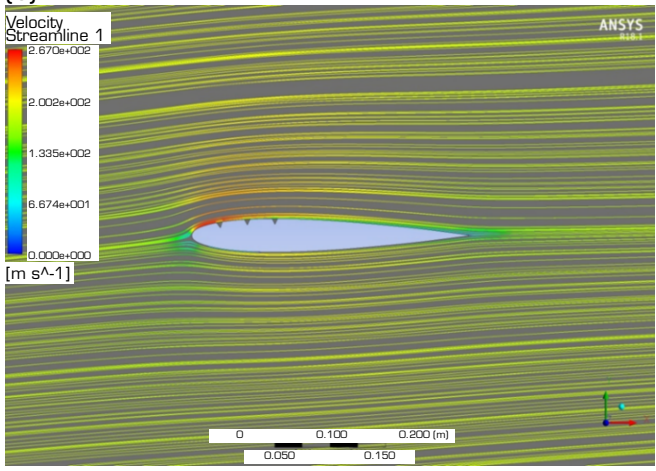

(d)

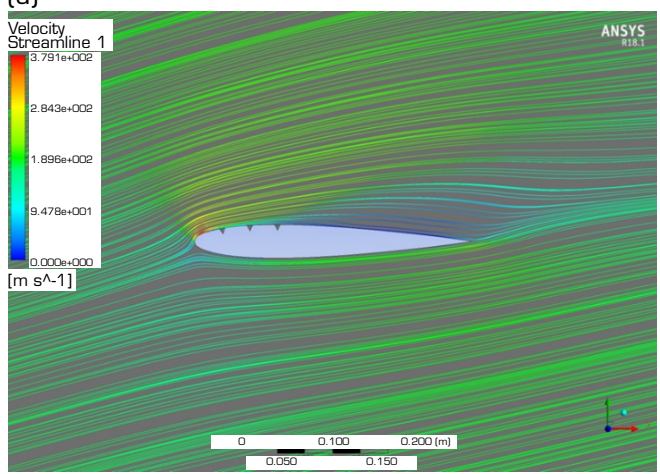

Figure 25. Streamlines for design 4 at (a) $0^{\circ}$, (b) $5^{\circ}$, (c) $10^{\circ}$ and (d) $15^{\circ}$.

(a)

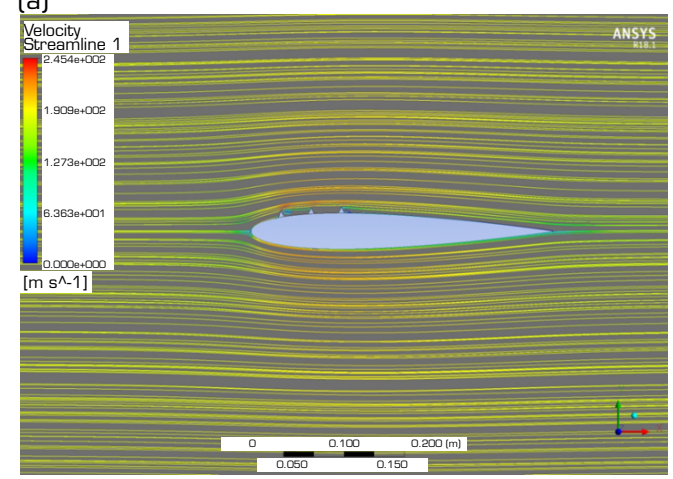

(c)

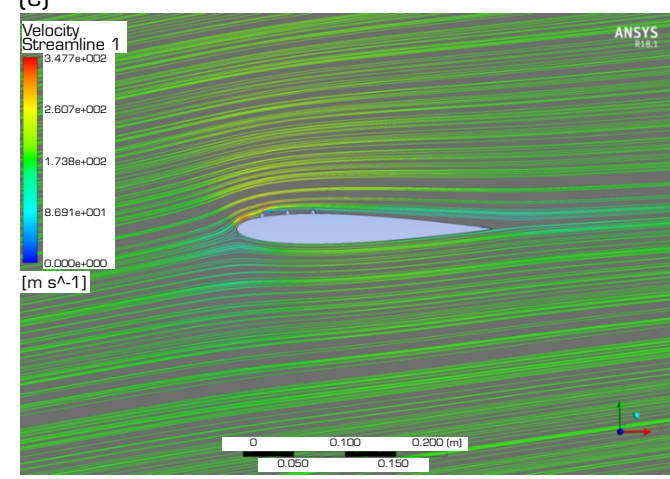

(b)

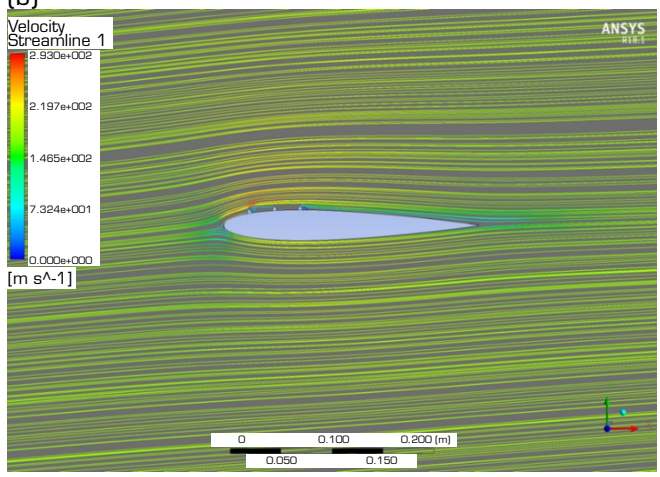

(d)

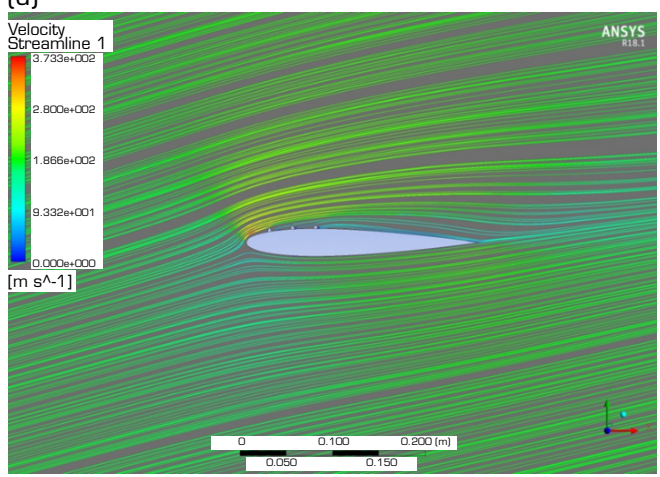

Figure 26. Streamlines for design 5 at (a) $0^{\circ}$, (b) $5^{\circ}$, (c) $10^{\circ}$ and (d) $15^{\circ}$. 
(a)

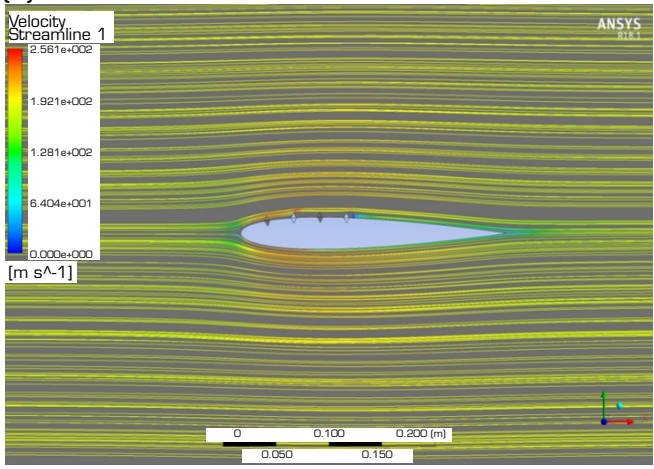

(c)

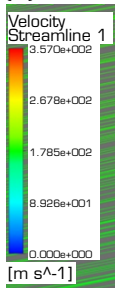

(b)

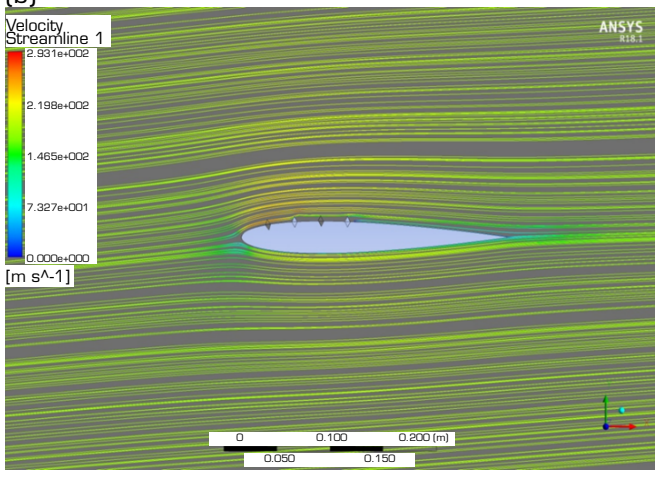

(d)

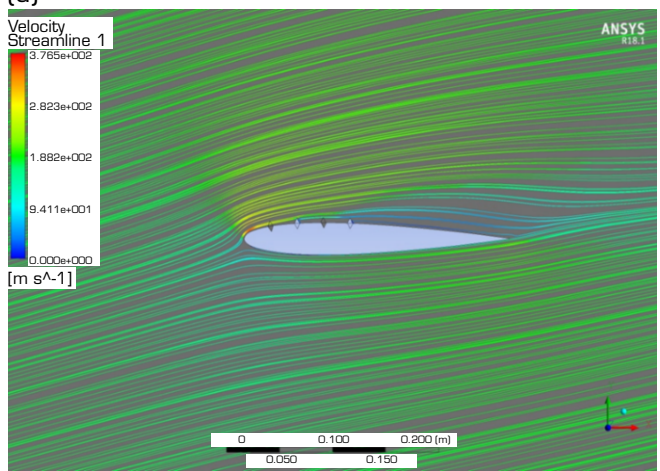

Figure 27. Streamlines for design 6 for the plane at $0.125 \mathrm{~m}$ at (a) $0^{\circ}$, (b) $5^{\circ}$, (c) $10^{\circ}$ and (d) $15^{\circ}$.

(a)

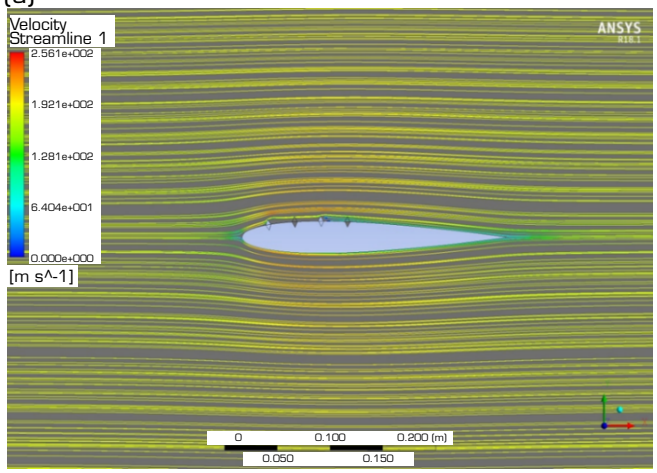

(c)

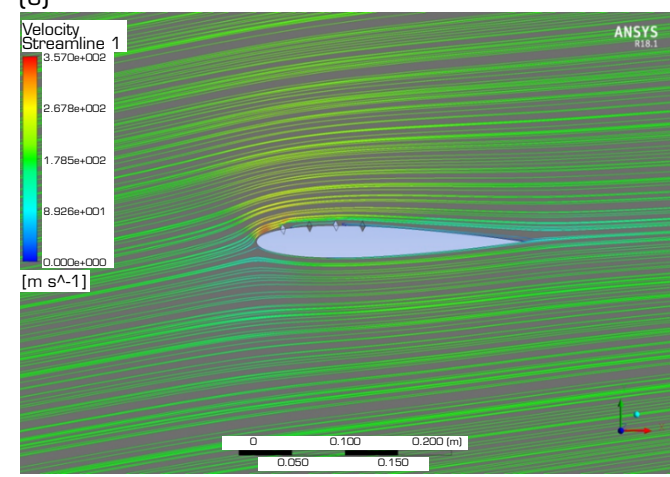

(b)

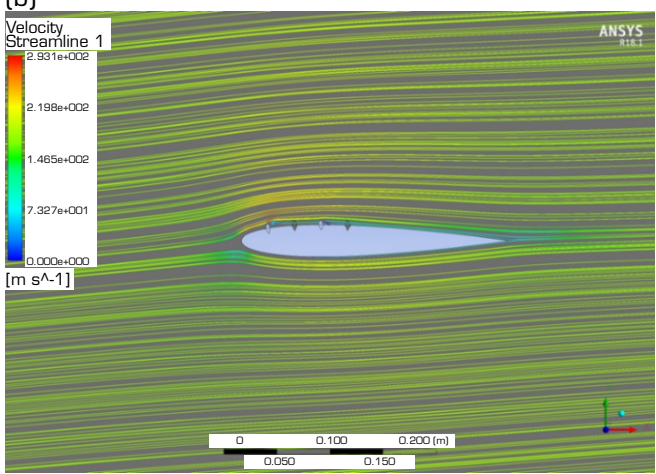

(d)

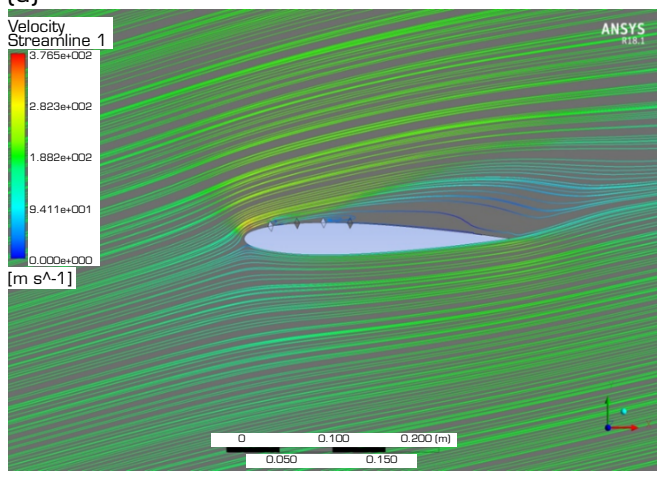

Figure 28. Streamlines for design 6 for the plane at $0.15 \mathrm{~m}$ at (a) $0^{\circ}$, (b) $5^{\circ}$, (c) $10^{\circ}$ and (d) $15^{\circ}$. 
The contours and streamlines are also compared to understand the effect of the concavity and shape of the feature. This is done by comparing:

1. Design 1 (Figs. 12 and 21) to design 2 (Figs. 13 and 22)

2. Design 4 (Figs. 16 and 25) to design 5 (Figs. 17 and 26)

3. Design 1 (Figs. 12 and 21) to design 4 (Figs. 16 and 25)

4. Design 2 (Figs. 13 and 22) to design 5 (Figs. 17 and 26)

From the comparisons, it can be inferred that spherical dimples and pyramidical bumps are better at preventing boundary layer separation when compared to their counterparts with different shapes and features.

The compound models with both dimples and bumps were also compared. First, for each shape (spherical and pyramidical), the results for the plane at $0.125 \mathrm{~m}$ are compared with the results for the plane at $0.15 \mathrm{~m}$. Then, for each plane, the corresponding spherical and pyramidical shapes are compared. This results in the following comparisons:

5. Design 3 at $0.125 \mathrm{~m}$ (Figs. 14 and 23) to design 3 at $0.15 \mathrm{~m}$ (Figs. 15 and 24)

6. Design 6 at $0.125 \mathrm{~m}$ (Figs. 18 and 27) to design 6 at $0.15 \mathrm{~m}$ (Figs. 19 and 28)

7. Design 3 at $0.125 \mathrm{~m}$ (Figs. 14 and 23) to design 6 at $0.125 \mathrm{~m}$ (Figs. 18 and 27)

8. Design 3 at $0.15 \mathrm{~m}$ (Figs. 15 and 24) to design 6 at $0.15 \mathrm{~m}$ (Figs. 19 and 28)

From the comparisons, it can be concluded that for compound designs, spherical features are better at delaying separation. It can also be concluded from comparisons (5) and (6) that when using a compound design, it is preferable to start the pattern sequence with a dimple rather than a bump. This also leads to the conclusion that the first feature has the most impact on the flow pattern.

\section{Coefficient of lift and drag}

The coefficient of lift and drag values are computed and the data is tabulated and plotted to understand the quantitative performance of the airfoil.

\section{Coefficient of lift}

The values of the coefficient of lift, $C_{i}$, are tabulated in Table 5 and are plotted as shown in Fig. 29.

Table 5. Coefficient of lift values for airfoil designs for varying AOA.

\begin{tabular}{|c|c|c|c|c|c|}
\hline \multicolumn{2}{|c|}{$\mathrm{AOA} \rightarrow$} & $0^{\circ}$ & $5^{\circ}$ & $10^{\circ}$ & $15^{\circ}$ \\
\hline \multirow{7}{*}{$\begin{array}{l}\text { Coefficient of } \\
\text { lift, } C_{l}\end{array}$} & Clean & -0.0001099 & 0.2495712 & 0.51226743 & 0.55347454 \\
\hline & Design 1 & -0.0017678 & 0.25109871 & 0.516168 & 0.53828473 \\
\hline & Design 2 & -0.0077496 & 0.23412341 & 0.47743536 & 0.51047907 \\
\hline & Design 3 & -0.0061816 & 0.23925518 & 0.49051929 & 0.52332599 \\
\hline & Design 4 & -0.002229 & 0.24741497 & 0.50915699 & 0.51274893 \\
\hline & Design 5 & -0.0162959 & 0.21967058 & 0.45230978 & 0.5036865 \\
\hline & Design 6 & -0.0150071 & 0.22291421 & 0.46597468 & 0.51342392 \\
\hline
\end{tabular}

The curves and the tabulated data in Table 5 indicate that the clean airfoil has the highest lift coefficient $\left(C_{l}\right)$ curve and has the highest lift at $15^{\circ}$, while design 5 has the lowest lift at $15^{\circ}$ with also the lowest lift curve out of all the designs. Design 1 has the highest lift curve when compared to other airfoils with surface modifications and is very similar to the curve of the clean airfoil. Design 1 is followed by design 3, design 6, design 4, and design 2.

When comparing the models with each other, it can be concluded that designs with dimples have a higher lift coefficient than the designs with only bumps for both spherical and pyramidical surface modifications. When comparing the shape of the modification, spherical shapes were found to have a better lift performance than pyramidical shapes. 


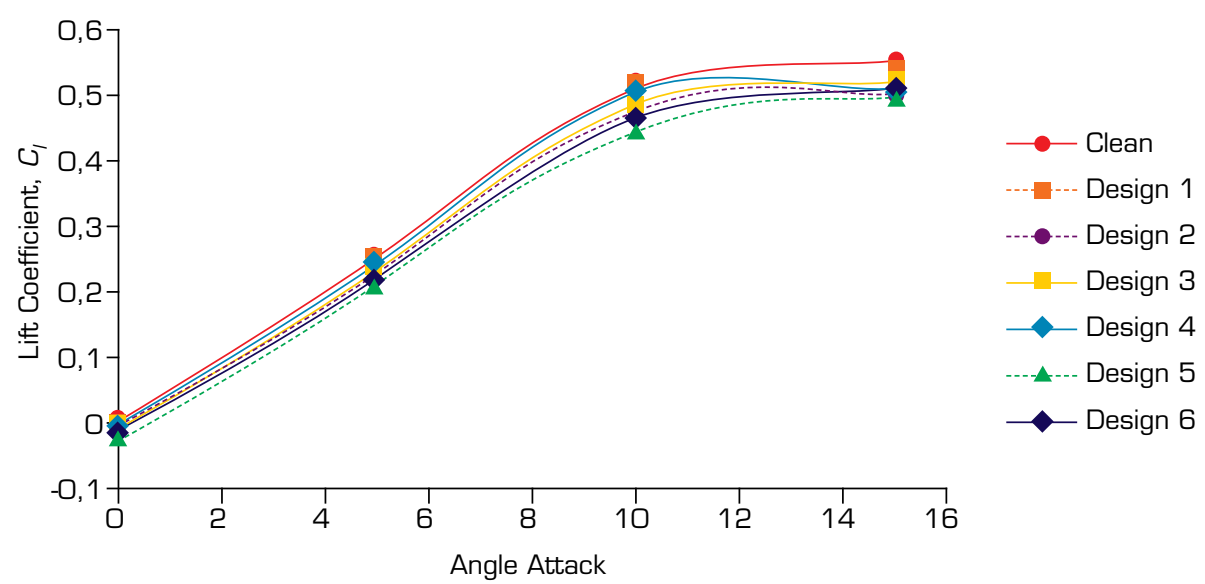

Figure 29. Comparison of lift coefficients for varying AOA.

Table 6 shows the percentage change in the $C_{l}$ values for models with surface modifications from the initial clean airfoil for 5,10 and $15^{\circ}$.

Table 6. Percentage change in $C_{l}$ values.

\begin{tabular}{cccccccc}
\hline AOA & Design 1 (\%) & Design 2 (\%) & Design 3 (\%) & Design 4 (\%) & Design 5 (\%) & Design 6 (\%) \\
\hline $5^{\circ}$ & 0.612 & -6.190 & -4.133 & -0.864 & -11.981 & -10.681 \\
\hline $10^{\circ}$ & 0.761 & -6.800 & -4.245 & -0.607 & -11.704 & -9.037 \\
\hline $15^{\circ}$ & -2.744 & -7.768 & -5.447 & -7.358 & -8.996 & -7.236 \\
\hline
\end{tabular}

From the data in Table 6, it is apparent that all models experience a negative change in the lift for $15^{\circ}$. Design 1 is the only model which experiences a positive change in the lift coefficient at 5 and $10^{\circ}$. This proves that spherical dimples improve lift performance for lower AOAs and their inclusion can contribute to better lift during take-off. For $15^{\circ}$, however, the smooth airfoil is the best design.

\section{Coefficient of drag}

The values of the coefficient of drag, $C_{d}$, are tabulated in Table 7 and are plotted as shown in Fig. 30.

Table 7. Coefficient of drag values for airfoil designs for varying AOA.

\begin{tabular}{cccccc}
\hline \multicolumn{2}{c}{ AOA $\rightarrow$} & $\mathbf{0}^{\circ}$ & $\mathbf{5}^{\circ}$ & $\mathbf{1 0}^{\circ}$ & $\mathbf{1 5}^{\circ}$ \\
\hline & Clean & 0.01303133 & 0.02393049 & 0.05944249 & 0.15052348 \\
\cline { 2 - 6 } & Design 1 & 0.011132 & 0.02197756 & 0.05723051 & 0.13086211 \\
\cline { 2 - 6 } & Design 2 & 0.01491537 & 0.0255637 & 0.05898553 & 0.13255562 \\
\cline { 2 - 6 } $\begin{array}{c}\text { Coefficient of } \\
\text { drag, } C_{d}\end{array}$ & Design 3 & 0.01445739 & 0.02506294 & 0.05873704 & 0.13352368 \\
\cline { 2 - 6 } & Design 4 & 0.01321999 & 0.02387334 & 0.05870769 & 0.13698986 \\
\cline { 2 - 6 } & Design 5 & 0.01404654 & 0.02442245 & 0.05756796 & 0.13797766 \\
\hline & Design 6 & 0.01728775 & 0.02775284 & 0.06096682 & 0.13525001
\end{tabular}




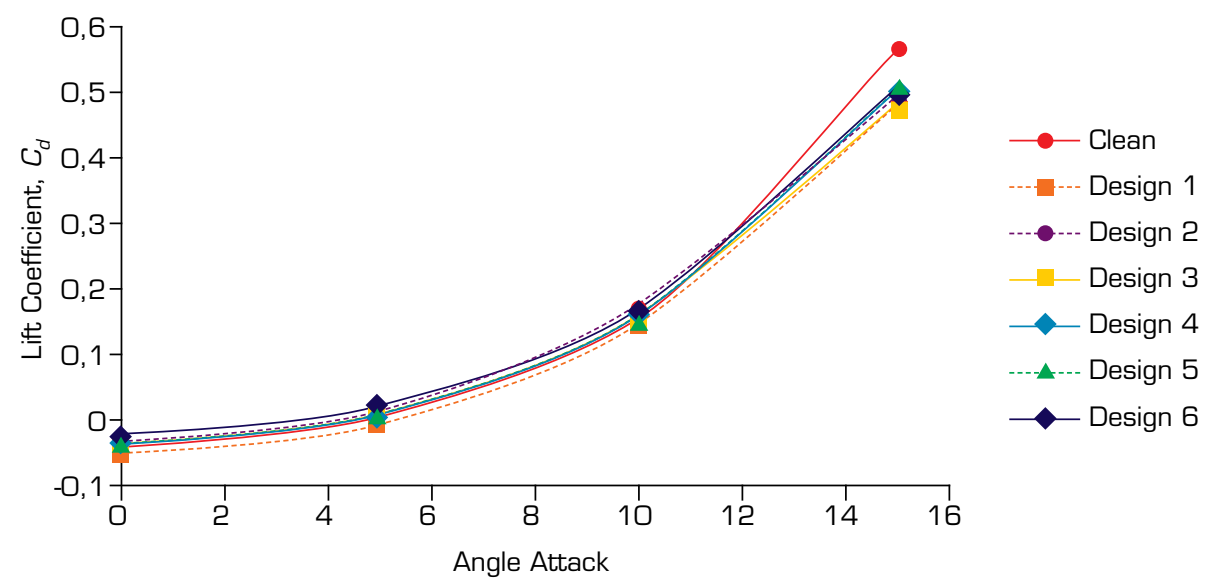

Figure 30. Comparison of drag coefficients for varying AOA.

The curves and the tabulated data in Table 7 indicate that the clean airfoil has the highest drag coefficient $\left(C_{d}\right)$ curve and has the highest drag at $15^{\circ}$, while design 1 has the lowest drag. All surface modifications contributed to a lower drag coefficient. After design 1, design 2 has the lowest drag followed by design 3, design 6, design 4 and then design 5.

After comparing all the models, it can be concluded that all surface modifications decreased the drag experienced by the airfoil. Pyramidical features experienced a greater drag than their spherical counterparts. Also, for both spherical and pyramidical features, dimples performed better than bumps in terms of the drag.

Table 8 shows the percentage change in the $C_{d}$ values for models with surface modifications from the initial clean airfoil for 5,10 and $15^{\circ}$.

Table 8. Percentage change in $C_{d}$ values.

\begin{tabular}{ccccccc}
\hline AOA & Design 1 [\%] & Design 2 (\%) & Design 3 (\%) & Design 4 (\%) & Design 5 [\%) & Design 6 [\%] \\
\hline $5^{\circ}$ & -8.161 & 6.825 & 4.732 & -0.239 & 2.056 & 15.972 \\
\hline $10^{\circ}$ & -3.721 & -0.769 & -1.187 & -1.236 & -3.154 & 2.564 \\
\hline $15^{\circ}$ & -13.062 & -11.937 & -11.294 & -8.991 & -8.335 & -10.147 \\
\hline
\end{tabular}

From the data in Table 8 , it is apparent that all models experience a negative change in the drag at $15^{\circ}$. Models with only dimples, design 1 and design 4, experience a negative change in $C_{d}$ at all AOAs. This leads to the conclusion that all design modifications lead to a reduction in drag at $15^{\circ}$, while dimples are more effective at reducing drag for all AOAs.

\section{Lift-to-drag ratio performance}

The values of the lift-to-drag ratio, $L / D$, are tabulated in Table 9 and are plotted, as shown in Fig. 31 .

The curves and the tabulated data in Table 9 indicate that for $15^{\circ}$, design 1 has the highest $\mathrm{L} / \mathrm{D}$, while design 5 has the lowest $\mathrm{L} / \mathrm{D}$, even lower than the clean airfoil. After design 1, design 3 has the highest L/D followed by design 2, design 6, design 4 and then the clean design.

After comparing all the models, it can be concluded that all surface modifications, except pyramidical bumps, increased the performance of the airfoil. The design with pyramidical protrusions, design 5, performed poorly compared with the clean design. Spherical features performed better than their pyramidical counterparts. Also, dimples, in general performed better than bumps for both types of shapes.

Table 10 shows the percentage change in the $\mathrm{L} / \mathrm{D}$ values for models with surface modifications from the initial clean airfoil for 5,10 and $15^{\circ}$. 
Table 9. Lift-to-drag ratio values for airfoil designs for varying AOA.

\begin{tabular}{cccccc}
\hline \multicolumn{2}{c}{ AOA $\rightarrow$} & $\mathbf{0}^{\circ}$ & $\mathbf{5}^{\circ}$ & $10^{\circ}$ & $\mathbf{1 5}^{\circ}$ \\
\hline & Clean & -0.0084347 & 10.4290054 & 8.61786613 & 3.67699803 \\
\cline { 2 - 6 } & Design 1 & -0.1588034 & 11.4252315 & 9.01910521 & 4.11337346 \\
\cline { 2 - 6 } & Design 2 & -0.5195725 & 9.15843351 & 8.09411027 & 3.85105565 \\
\cline { 2 - 6 } $\begin{array}{c}\text { Lift-to-drag } \\
\text { ratio } L / D\end{array}$ & Design 3 & -0.4275709 & 9.54617528 & 8.35110616 & 3.91934966 \\
\cline { 2 - 6 } & Design 4 & -0.1686085 & 10.3636513 & 8.67274766 & 3.74296995 \\
\cline { 2 - 6 } & Design 5 & -1.1601345 & 8.99461684 & 7.85697051 & 3.65049313 \\
\cline { 2 - 6 } & Design 6 & -0.8680744 & 8.03212277 & 7.64308614 & 3.79611003 \\
\hline
\end{tabular}

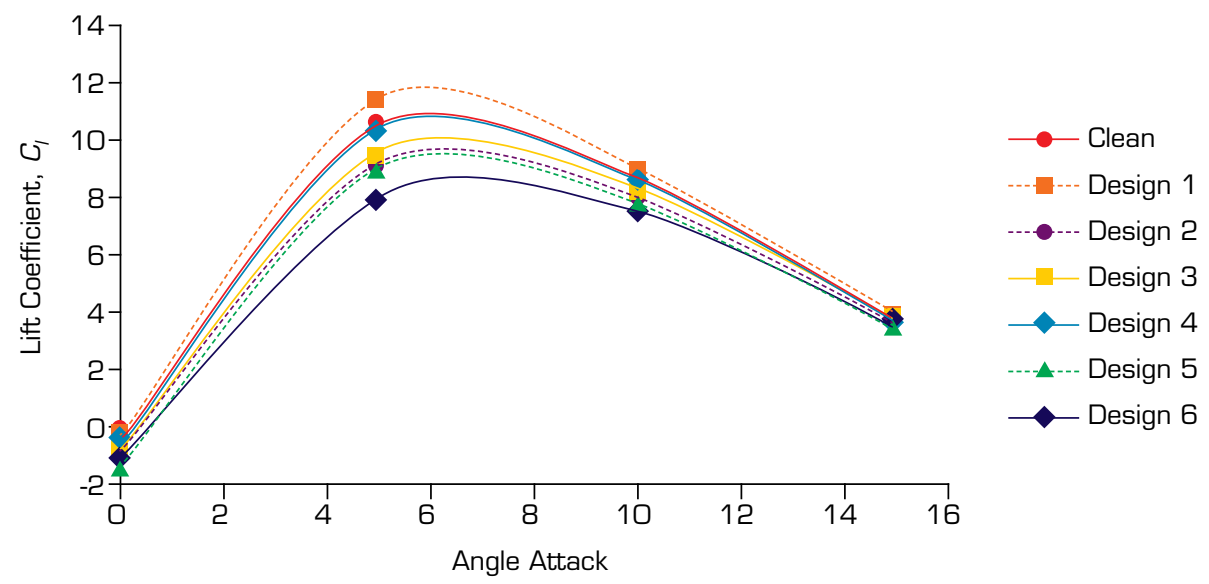

Figure 31. Comparison of lift-to-drag for varying AOA.

Table 10. Percentage change in $L / D$ values.

\begin{tabular}{ccccccc}
\hline AOA & Design 1 (\%) & Design 2 (\%) & Design 3 (\%) & Design 4 (\%) & Design 5 (\%) & Design 6 (\%) \\
\hline $5^{\circ}$ & 9.552 & -12.183 & -8.465 & -0.627 & -13.754 & -22.983 \\
\hline $10^{\circ}$ & 4.656 & -6.078 & -3.095 & 0.637 & -8.829 & -11.311 \\
\hline $15^{\circ}$ & 11.868 & 4.734 & 6.591 & 1.794 & -0.721 & 3.239 \\
\hline
\end{tabular}

From the data in Table 10, it is apparent that all models, with the exception of design 5, experience an increase in L/D performance at $15^{\circ}$. Design 5 , in fact, has a lower performance than the clean airfoil. The design with the greatest increase in performance is design 1 , which experiences not only the greatest performance at $15^{\circ}$, but also is the only design to experience an increase in the performance at all AOAs. After design 1, the best performing design in terms of increase in performance for a wider range of AOAs is design 4 , which has an increase in performance for 10 and $15^{\circ}$. Also, it can be observed that design 1, design 2 , and design 3 are better performing than their pyramidical counterparts at $15^{\circ}$ as they, in general, experience a lower decrease for other AOAs.

After analyzing and discussing all the results, it can be concluded that design 1 is the best performing airfoil design in terms of flow visualization and L/D performance. From Figs. 20 and 21, there is a significant improvement in the boundary layer separation from the clean airfoil. This improvement is also accompanied with an improvement in the $\mathrm{L} / \mathrm{D}$ performance. Therefore, spherical dimples are the best design modification to delay flow separation and improve performance. 
Design 5, although shows promising results from the streamline and velocity plots, fails to provide quantitative improvement in the performance with a L/D performance ratio lower than the clean airfoil at higher AOA.

In general, spherical designs performed better than pyramidical designs and dimples were more adept at increasing performance for both spherical and pyramidical designs.

\section{Comparison of lift, drag and performance with other techniques}

To further understand the trends that surface modifications have on the aerodynamic performance of an airfoil, the results of the current study are compared with the findings of other similar studies and tabulated in Table 11 . The findings are presented as percentage changes in the $C_{l}, C_{d}$ and $\mathrm{L} / \mathrm{D}$ values from the baseline smooth airfoil. For some of the values, the percentage changes had to be calculated, as the authors did not present their findings in the form of percentage changes.

Table 11. Comparison with other surface modification studies.

\begin{tabular}{|c|c|c|c|c|}
\hline Authors & Surface modification & Change in $C_{l}$ & Change in $C_{d}$ & Change in $L / D$ \\
\hline Current study & $\begin{array}{l}\text { Patterns of spherical } \\
\text { and pyramid dimples } \\
\text { and bumps }\end{array}$ & $\begin{array}{l}3 \% \text { decrease for design } \\
\text { with dimples for } 15^{\circ}, \\
\text { improved performance } \\
\text { at lower AOA }\end{array}$ & $\begin{array}{c}13 \% \text { decrease for } \\
\text { design with dimples for } \\
15^{\circ}\end{array}$ & $\begin{array}{l}12 \% \text { increase for design } \\
\text { with dimples for } 15^{\circ}\end{array}$ \\
\hline Saraf et al. (2017) & $\begin{array}{l}\text { Spherical dimples at } \\
10,25,50 \text { and } 75 \% \text { of } \\
\text { chord }\end{array}$ & $\begin{array}{l}7 \% \text { increase for dimple } \\
\text { at } 75 \% \text { chord for } 16^{\circ}\end{array}$ & $\begin{array}{l}3 \% \text { decrease for dimple } \\
\text { at } 75 \% \text { chord for } 16^{\circ}\end{array}$ & $\begin{array}{l}6 \% \text { increase for dimple } \\
\text { at } 75 \% \text { chord for } 16^{\circ}\end{array}$ \\
\hline $\begin{array}{l}\text { Mustak et al. } \\
\text { [2017] }\end{array}$ & $\begin{array}{c}\text { One row of hexagonal } \\
\text { bumps }\end{array}$ & $\begin{array}{c}\text { Around } 33.3 \% \text { increase } \\
\text { for } 16^{\circ}\end{array}$ & $\begin{array}{l}\text { Around } 7.46 \% \\
\text { decrease for } 16^{\circ}\end{array}$ & $\begin{array}{c}\text { Around } 31.1 \% \text { increase } \\
\text { for } 16^{\circ}\end{array}$ \\
\hline $\begin{array}{l}\text { Mustak et al. } \\
\text { [2015] }\end{array}$ & $\begin{array}{l}\text { Row of dimples and } \\
\text { bumps }\end{array}$ & $\begin{array}{l}\text { Around } 38.6 \% \text { increase } \\
\text { for dimple for } 16^{\circ}\end{array}$ & $\begin{array}{l}\text { Around } 7.46 \% \text { increase } \\
\text { for dimple for } 16^{\circ}\end{array}$ & $\begin{array}{l}\text { Around } 29.3 \% \text { increase } \\
\text { for dimple for } 16^{\circ}\end{array}$ \\
\hline $\begin{array}{c}\text { Zulkefli and } \\
\text { MohdNur (2020) }\end{array}$ & $\begin{array}{l}\text { Row of inboard dimple, } \\
\text { outboard dimple, and } \\
\text { triangular VG at } 50 \% \\
\text { chord }\end{array}$ & $\begin{array}{l}\text { Around } 14.7 \% \text { increase } \\
\text { for triangular VG and } R e \\
100,000 \text { for } 16^{\circ}\end{array}$ & $\begin{array}{c}\text { Around } 38.4 \% \\
\text { decrease for triangular } \\
\text { VG and } R e 100,000 \\
\text { for } 16^{\circ}\end{array}$ & $\begin{array}{l}\text { Around } 84.8 \% \text { increase } \\
\text { for triangular VG and } R e \\
100,000 \text { for } 16^{\circ}\end{array}$ \\
\hline
\end{tabular}

Saraf et al. (2017) analyzed a NACA 0012 airfoil that was altered by dimples. They conducted a CFD analysis for dimples located at four different positions (10,25, 50 and 75\% of chord length) along the airfoil to understand the effect of the dimples location on the performance. The study was conducted on varying AOA from 0 to $16^{\circ}$.

Zulkefli and MohdNur (2020) studied the effect of an inboard dimple, an outboard dimple, and a triangular vortex generator at $50 \%$ chord length on the flow around a NACA 4415 airfoil. They conducted the CFD analysis for the airfoil models for Re of 50000 and 100000 at varying AOA. They found an improved performance for all modifications. However, they found that the lift is highest for a triangular vortex generator while the drag is lowest for the inward dimple and triangular vortex generator. The inward dimple has the best $\mathrm{L} / \mathrm{D}$ performance from all modifications.

Mustak et al. (2015) studied the effect of dimples and bumps on the performance of a NACA 4415 airfoil. They found that the separation of flow occurs at $12^{\circ}$ for a smooth airfoil but occurs at around $16^{\circ}$ for the modified airfoils. They also found that both textured airfoils increased lift when compared to the smooth one for all AOA.

Mustak et al. (2017) studied the effect of hexagonal protrusions on the aerodynamic efficiency of a NACA 4415 airfoil. The flow was studied in a wind tunnel at subsonic airspeeds for AOA of 0 to $18^{\circ}$. It was found that for the smooth airfoil, the flow separated at $12^{\circ}$ but for the textured airfoil, it separated at $16^{\circ}$. The airfoil with bumps also shows a $19.3 \%$ increase in lift, a $48.39 \%$ reduction in drag and an overall $53.75 \%$ increase in $\mathrm{L} / \mathrm{D}$ performance.

The data in Table 11 is presented to understand the effects on lift, drag and performance at a similar AOA. From the data in Table 8, all studies except the current one reported an increase in lift at a similar AOA. However, Mustak et al. (2015) also reported an increase in drag for $16^{\circ}$. All studies reported an increase in $\mathrm{L} / \mathrm{D}$ performance at the given AOA. Therefore, it can be concluded that, for the most part, the introduction of surface features leads to an increase in the aerodynamic performance of an airfoil along with an increase in the lift and a reduction in drag. 


\section{CONCLUSION}

The aim of this study was to understand the airflow around an airfoil and how the introduction of surface features like dimples and bumps affect the flow. The CFD analysis of six design features on a base airfoil of NACA 0012 was conducted for AOA of 0 to $15^{\circ}$. From the results, the following conclusions can be made:

- All modifications result in an improved flow alignment and delayed boundary layer separation. For a clean NACA 0012 airfoil, the flow separates and recirculates at $15^{\circ}$. For the airfoils with texture, however, the flow does not recirculate and remains attached for longer, as evident from the streamlines.

- Most design modifications, with the exception of pyramidical bumps, led to an increase in the L/D performance.

- Spherical dimples (design 1) have the best design performance of all other design modifications. From Figs. 20 and 21 , there is a significant improvement in the boundary layer separation from the clean airfoil. This improvement is also accompanied with an improvement in the L/D performance. Therefore, spherical dimples are the best design modification to delay flow separation and improve performance. Spherical dimples create tiny vortices in their concavities, which cause the boundary layer to become turbulent and remain attached for longer.

- Although pyramidical bumps (design 5) have better flow alignment, as evident from the streamline contours when compared to pyramidical dimples (design 4) and spherical bumps (design 2), they are the only design to experience a reduction in performance; one which is even lower than the clean airfoil. However, the combination of pyramidical dimples and bumps, as seen in design 6 , causes the performance to be greater than the designs with only one type of feature.

- When comparing the type of shape, i.e., either spherical or pyramidical, it can be concluded that spherical shapes are more ideal.

- It can also be concluded that when considering the type of feature, for both types of shapes, dimples are far superior to bumps.

\section{AUTHORS' CONTRIBUTION}

Conceptualization: Mehtar Z; Formal Analysis: Mehtar Z; Investigation: Mehtar Z; Methodology: Mehtar Z; Project Administration: Mehtar Z and Altaf A; Resources: Mehtar Z and Altaf A; Supervision: Altaf A; Validation: Mehtar Z and Altaf A; Visualization: Mehtar Z and Altaf A; Writing - Original Draft Preparation: Mehtar Z; Writing - Review and Editing: Altaf A.

\section{DATA AVAILABILITY STATEMENT}

All data sets were generated or analyzed in the current study.

\section{FUNDING}

Not Applicable.

\section{ACKNOWLEDGEMENTS}

Not applicable. 


\section{REFERENCES}

Baweja C, Dhannarapu R, Niroula U, Prakash I (2016) Analysis and optimization of dimpled surface modified for wing planforms. Paper presented 7th International Conference on Mechanical and Aerospace Engineering (ICMAE). IEEE; London, United Kingdom. https://doi.org/10.1109/ICMAE.2016.7549590

Bogdanović-Jovanović, Stamenković Z, JB, Kocić M (2012) Experimental and numerical investigation of flow around a sphere with dimples for various flow regimes. Therm Sci 16(4):1013-1026. https://doi.org/10.2298/TSCI120412115B

Chalia S, Bharti M (2017) Design and Analysis of Vortex Generator and Dimple over an Airfoil Surface to Improve Aircraft Performance. IJA-ERA 3(4):173:181.

Chear CK, Dol SS (2015) Vehicle Aerodynamics: Drag Reduction by Surface Dimples. Int J Mech Mechatron Eng 9(1):202-205.

Dhiliban A, Meena P, Narasimhan P, Vivek M (2013) Aerodynamic Performance of Rear Roughness Aerofoils. Paper presented 8th Asia-Pacific Conference on Wind Engineering. Chennai, India. https://doi.org/10.3850/978-981-07-8012-8_252

Faruqui SHA, Al Bari A, Emran AF (2014) Numerical Analysis of Role of Bumpy Surface to Control the Flow Separation of an Airfoil. Procedia Eng 90:255-260. https://doi.org/10.1016/j.proeng.2014.11.846

Hong S, Asai T (2017) Aerodynamic effects of dimples on soccer ball surfaces. Heliyon 3(10):e00432. https://doi.org/10.1016/j. heliyon.2017.e00432

Hornea MV, Simion I (2019) Using Perturbed Airflow Surfaces to Reduce Drag. J Ind Design Eng Graphics 14(1):243-248.

Kalkur A (2017) Enhancement of Aerodynamics of Airfoils by Surface Modifications - Numerical Study. Int J Sci Eng Dev Res 2(8):169-173.

Livya E, Anitha P, Valli P (2015) Aerodynamic Analysis of Dimple Effect on Aircraft Wing. International Journal of Mechanical, Aerospace, Industrial, Mechatronic and Manufacturing Engineering 9(2):350-353.

Mustak R, Molla H-O-R, Mashud M (2017) Improvement of aerodynamic characteristics of an airfoil by surface modification. Am J Eng Res 6(3):7-14.

Mustak R, Uddin N, Mashud M (2015) Effect of Different Shaped Dimples on Airfoils. Paper presented 3rd International Conference on Mechanical Engineering and Renewable Energy. Chittagong, Bangladesh.

Ramprasadh C, Devanandh V (2015) A CFD Study on Leading Edge Wing Surface Modification of a Low Aspect Ratio Flying Wing to Improve Lift Performance. Int J Micro Air Veh 7(3):361-373. https://doi.org/10.1260/1756-8293.7.3.361

Saraf AK, Singh MP, Chouhan TS (2017) Effect of Dimple on Aerodynamic Behaviour of Airfoil. Int J Eng Technol 9(3):22682277. https://doi.org/10.21817/ijet/2017/v9i3/1709030335

Srivastav D (2012) Flow control over airfoils using different shaped dimples. In: International Conference on Fluid Dynamics and Thermodynamics Technologies. Singapore: IACSIT. p. 92-97.

Zulkefli N, MohdNur N (2020) Dimples and Vortex Generator Performance on Airfoil Surface. Int J Adv Sci Technol 29(6):208-213. 